\title{
Les enjeux esthétiques du jeu vidéo : entre art, stylistique et interactivité
}

\section{Martin Picard}

\section{(2) OpenEdition}

\section{Journals}

Édition électronique

URL : http://journals.openedition.org/sdj/712

DOI : $10.4000 /$ sdj. 712

ISSN : 2269-2657

\section{Éditeur}

Laboratoire EXPERICE - Centre de Recherche Interuniversitaire Expérience Ressources Culturelles Education

\section{Référence électronique}

Martin Picard, «Les enjeux esthétiques du jeu vidéo : entre art, stylistique et interactivité », Sciences du jeu [En ligne], 6 | 2016, mis en ligne le 18 octobre 2016, consulté le 19 avril 2019. URL : http:// journals.openedition.org/sdj/712 ; DOI : 10.4000/sdj.712

Ce document a été généré automatiquement le 19 avril 2019.

Tous droits réservés 


\title{
Les enjeux esthétiques du jeu vidéo : entre art, stylistique et interactivité
}

\author{
Martin Picard
}

\section{Jeu vidéo et théorie esthétique}

1 En 1998, Espen Aarseth mentionnait déjà que le jeu vidéo représentait « un nouveau mode d'esthétique, ainsi que de discours social, une alternative au récit qui a été le paradigme dominant jusqu'à présent : le principal moyen de transmettre des connaissances et une expérience » (Aarseth, 1998 ; ma traduction). Même si on a fréquemment signalé ses affinités avec le cinéma (King et Krzywinska, 2002 ; Perron et Therrien, 2009), l'expérience vidéoludique est aussi caractérisée autant par la capacité de l'ordinateur à simuler un espace que par le contrôle qui est donné au joueur à déterminer sa direction et son action. Malgré le fait que ce contrôle et cette présence soient limités, cette "liberté » d'action, même partielle, permet néanmoins d'accroître certaines sensations d'immersion. Le désir de réalisme, dans le sens d'une exactitude de perception sensorielle, est souvent central aux pratiques discursives sur le jeu vidéo, principalement dans les campagnes de marketing de nouvelles consoles ou de nouveaux jeux triple-A, mais aussi dans le prolongement de ces discours dans les presses généralistes et spécialisées, de même que dans certaines communautés de joueurs. Une sensation participative et un réalisme spectaculaire (Darley, 2000) dépendent en effet de l'amélioration constante des représentations visuelles. Représentations visuelles et esthétiques semblent ainsi souvent se confondre lorsque l'on se penche sur les qualités artistiques du jeu vidéo.

Néanmoins, la question se pose tout de même : existe-t-il une « esthétique » spécifique au jeu vidéo ? La littérature sur le sujet dans les études sur le jeu vidéo nous permet déjà de répondre par l'affirmative (Grodal, 2003; Egenfeldt-Nielsen, Smith et Tosca, 2008, p. 97-131; Lundgren, Bergström et Björk, 2009 ; Niedenthal, 2008, 2009 ; Sommerseth, 2009 ; Tavinor, 2009). Par contre, en abordant la question sous la perspective de la philosophie 
de l'art, la mauvaise presse qu'a la notion d'esthétique depuis une quarantaine d'années (un certain renouveau s'est tout de même imposé) ${ }^{1}$ nuit à l'intérêt que devrait avoir une telle question.

3 Cependant, dans le cadre d'une réflexion sur la relation entre le jeu vidéo et l'art, l'élaboration d'une esthétique du jeu vidéo - même rapide et incomplète comme celle proposée ici - prend une importance cruciale étant donné que celle-ci est directement liée à l'interaction entre le joueur et le jeu. L'esthétique a dans le jeu vidéo une fonction structurante (Egenfeldt-Nielsen, Smith et Tosca, 2008, p. 97), en plus des fonctions affective et artistique qu'elle partage avec les autres médias. Comme le mentionne Steven Poole, « [...] la réponse du joueur à un jeu bien conçu est en partie la même que pour un film ou une peinture, elle est esthétique [c'est l'auteur qui souligne] » (Poole, 2000, p. 29 ; ma traduction).

4 Bruno Samper, dans son texte " Les artistes et le jeu vidéo " qui s'inscrit dans une démarche de création de jeu (ici, le jeu Society), avance même l'idée que l'esthétique du jeu vidéo revêt une dimension métaphysique, car le jeu vidéo « [...] est une exploration du sensible, un questionnement de la notion de réalité " (Samper, 2003), à savoir fondamentalement une exploration ou une conquête d'un espace et/ou d'une altérité ${ }^{2}$. Selon Samper, cette ambition métaphysique se remarque par la mise en scène d'une quête, et par une représentation du sensible qui "révèle l'indicible ou l'invisible " (Samper, 2003). Il existe deux manières de représenter l'indicible dans le jeu vidéo : en creux (ou négatif), par la quête du (photo)réalisme où l'invisible est révélé en imitant au mieux le visible; ou en plein, où l'invisible est révélé en lui donnant une forme, que ce soit par la forme de créatures et de monstres (Samper donne pertinemment comme exemple les créatures apparentées aux poupées de Hans Bellmer dans Silent Hill 2 [Konami, 2001]), ou bien en prenant la forme d'une métaphore d'un monde parallèle, telles les zones secrètes (« warp zones ») de Super Mario Bros (Nintendo, 1985).

Puisqu'« une prospection métaphysique trouve ses moyens dans le choix et l'élaboration de systèmes de représentation du monde » (Samper, 2003), la dimension métaphysique du jeu vidéo mène nécessairement à sa dimension esthétique. La tâche principale ici est alors de tenter d'articuler une première réflexion sur une possible spécificité de l'esthétique du jeu vidéo, mais surtout en lien avec la relation ambivalente entre l'art et le jeu vidéo.

6 A priori, il est évident que ladite esthétique relève de l'esthétique générale, du discours philosophique sur l'Art dans son ensemble. Le point de départ serait le même que celui d'Aumont et alii (1994) pour le cinéma. En ce sens, l'esthétique du jeu vidéo « recouvre la réflexion sur les phénomènes de signification considérés en tant que phénomènes artistiques » (Aumont et alii, 1994, p. 7). Cependant, l'étude de l'esthétique du jeu vidéo ne pourrait reposer uniquement sur les réflexions philosophiques classiques de l'esthétique (Aristote [1990], Hegel [1953] ou Kant [1990 et 1995]). L'esthétique contemporaine, par son éclatement et ses ruptures, n'est plus une théorie autonome et homogène. L'esthétique du XXe siècle, et la pensée contemporaine en général (qu'on la qualifie de postmoderne ou non), nous a fait passer de l'homo(gène) à l'hétéro(gène), puis au multi (disciplinaire / médiatique / national) et finalement à l'inter (disciplinaire / médiatique / national). Depuis le XXI ${ }^{e}$ siècle, il y aurait eu dépassement de cette "postmodernité ». En ce temps de «cumul», selon la formule de Gervereau (2000), les relations sont désormais trans (disciplinaires / médiatiques / nationales). L'esthétique du jeu vidéo doit idéalement être une étude ou une réflexion transmédiale, transdisciplinaire, et transnationale, qui porte sur un objet actuel protéiforme et hybride, et qui souhaite 
souligner les phénomènes de significations considérés en tant que phénomènes artistiques.

7 Toutefois, même en souhaitant se distancer de l'association esthétique/science du Beau classique, l'étude de la question esthétique présuppose d'emblée qu'il y soit question d'art. Prétendre à une esthétique du jeu vidéo reviendrait à affirmer que le jeu vidéo soit un art. Cependant, dans le domaine du jeu vidéo, une telle affirmation est plus complexe qu'il n'y parait. Alors loin est mon intention ici d'entrer dans le sempiternel débat : « estce que le jeu vidéo est un art? $» .^{3}$ Ce débat montre la plupart du temps une méconnaissance de l'esthétique du jeu vidéo, soit par une confusion autour de la notion d'art elle-même, soit par omission de la prise en compte du caractère hybride et multidimensionnel du jeu vidéo. C'est pourquoi il est important d'éclaircir d'abord ce quiproquo tout en développant les enjeux esthétiques qui en découlent. Le jeu vidéo, considéré de façon générale dans son aspect protéiforme, comprend plusieurs niveaux artistiques. Ou, plus précisément, il peut être compris de différentes manières selon les conceptions de la notion d'art ou des multiples dimensions artistiques qui le caractérisent. Dès lors, dans ce qui suit, j'examinerai les différentes conceptions que peut prendre la notion d'art en lien avec le jeu vidéo, que je diviserai en trois grandes catégories: le jeu vidéo comme production artistique, comme média audiovisuel, et comme pratique interactive. Dans le cadre de mes réflexions sur son aspect médiatique, je préciserai l'importance des styles visuels du jeu vidéo, puisqu'ils contribuent de manière primordiale à sa perception en tant qu'objet esthétique.

\section{Le jeu vidéo comme technique artistique : la conception de jeux}

8 Le jeu vidéo est un art technique, en ce sens qu'il est du domaine du savoir-faire (crafting en anglais). De manière confuse, cette acception du terme " art » (comme en témoignent les nombreux ouvrages anglophones "The Art of ...») $)^{4}$ est, pour l'industrie, et plusieurs fans, suffisante pour conclure au caractère artistique du jeu vidéo. Cependant, cette utilisation ne doit pas être confondue avec la notion d'Art (que l'on a conventionnellement dénommé avec une lettre majuscule justement pour la différencier de l'acception commune) en usage dans les institutions artistiques. Cette dernière concerne principalement l'Histoire de l'Art et l'esthétique philosophique, dont les tentatives de définir cette notion ont marqué et accompagné l'histoire entière de la pensée occidentale. ${ }^{5}$

9 Comme le mentionne Laurent Jullier (1998) dans son ouvrage Les images de synthèse, « [1] 'institution artistique ne reconnait pas, bien entendu, la pertinence [du critère du savoir-faire]. Dès le début du siècle, les avant-gardes ont fait abandonner, pour juger de la valeur d'une œuvre d'art, [c]e critère ... » (Jullier, 1998, p. 79). Jullier remonte même à la conception de l'art chez Baudelaire pour exemplifier ce constat :

La poésie et le progrès, disait Baudelaire, sont deux ambitieux qui se haïssent d'une haine instinctive ; [ce dernier] se désolait qu'autour de lui « les yeux s'accoutument à considérer les résultats d'une science matérielle comme les produits du beau » (Jullier, 1998, p. 78).

Sébastien Genvo, quant à lui, ajoute que « la démonstration d'un savoir faire technique ne peut [...] à elle seule justifier la filiation d'un domaine avec une discipline artistique " (Genvo, 2003, p. 46). 
11 Pour l'industrie du jeu vidéo, le progrès et l'expertise techniques sont des aspects privilégiés, et ils sont souvent traités en tant que phénomènes artistiques. Cette conception souligne que les jeux vidéo sont d'abord et avant tout emblématiques d'une histoire du progrès technologique. Le matériel physique (hardware) et informatique ( software) du jeu vidéo, en constant développement, accroît les possibilités techniques et technologiques du jeu vidéo, principalement au niveau du «réalisme » des images. Le progrès technologique a des conséquences directes sur l'amélioration non seulement quantitative, mais aussi qualitative des images de synthèse et de l'animation numérique requises pour la création du jeu vidéo. Les possibilités créatrices sont directement associées aux possibilités technologiques du média. Les œuvres marquantes mentionnées dans les histoires du jeu vidéo (Space Invaders, Mario Bros., DOOM, etc.) sont souvent celles qui ont réussi à dépasser les limites que leur offrait le matériel technologique, et où les concepteurs ont su user d'originalité et de créativité en utilisant les contraintes à leurs avantages (Iwata asks, 2009 ; Bottomley, 2011). La "quincaillerie électronique» (le hardware) joue un rôle fondamental sur le caractère de l'œuvre vidéoludique. Elle dicte autant les possibilités de la jouabilité que l'aspect visuel des jeux.

En ce sens, «l'art » technique du jeu vidéo s'apparente au dessin animé traditionnel. La différence fondamentale réside dans l'outil utilisé, c'est-à-dire l'ordinateur dans le cas du jeu vidéo, entraînant du fait même des distinctions formelles et stylistiques. ${ }^{6}$ Plusieurs métiers sont sensiblement demeurés les mêmes (layout, concept art, animateur, intervalliste, etc.), alors que la chaîne de production s'est vue modifiée par l'ajout de nouvelles fonctions ou de nouveaux titres tels que le modeleur, le textureur, le programmeur, etc. La conception de jeu vidéo (le game design) repose également sur un ensemble d'expertises, d'habiletés et de savoirs-faire pratiques et techniques en plus de connaissances artistiques. Le concepteur de jeu (game designer) et le concepteur de niveaux (level designer) doivent porter plusieurs chapeaux à la fois. Ils doivent être : «metteur en jeu », par la création de la jouabilité qui requiert un talent dans la création d'un système de jeu interactif ; architecte, ${ }^{7}$ dans la création de l'univers vidéoludique en deux ou trois dimensions, du monde simulé dans lequel les joueurs navigueront ; programmeur, pour bien comprendre l'application informatique de tous les éléments d'un jeu vidéo ; et tout simplement artiste, dans la création d'une oeuvre signifiante, originale et émotionnelle. Comme le mentionne le concepteur de jeu Cliff Bleszinski :

Le level design est autant un art qu'une science ; il exige des compétences et un savoir-faire artistiques ainsi qu'une connaissance technique approfondie. Un concepteur avec une énorme expérience en art traditionnel ou en architecture ne réussira pas s'il ne peut pas comprendre des notions telles que le framerate, le gameflow, et le pacing. Un concepteur qui comprend ces éléments, mais qui n'a pas encore d'expérience en architecture ou en art est aussi voué à l'échec. Art et science sont le Yin et le Yang de la conception, et il faut l'effort d'individus très talentueux et dévoués pour produire des niveaux de haute qualité (Bleszinski, 2001; ma traduction).

\section{Le jeu vidéo comme média audiovisuel : sa relation avec les autres arts}

13 Le jeu vidéo n'est pas uniquement un art technique; c'est aussi, et surtout, un média audiovisuel. En ce sens, il est directement lié aux arts et industries culturelles qui se sont développés avant lui, tels que le cinéma, la photographie, l'animation et la bande 
dessinée. Ses matières d'expression proviennent essentiellement des autres arts, tandis que son langage se situe plutôt dans sa structure interactive spécifique. Ainsi, le jeu vidéo possède les mêmes prétentions artistiques dans ce domaine que les médias antérieurs (Genvo, 2003), offrant même une synthèse de ceux-ci à plusieurs degrés.

En s'appuyant sur le caractère narratif du cinéma, le jeu vidéo reprend de la littérature sa structure narrative et le développement de sa fiction. Une grande part des jeux vidéo ont des qualités narratives, que ce soit la mise en place d'un contexte ou d'un environnement ouvert à la manifestation d'évènements se déroulant dans un temps circonscrit, l'élaboration d'une intrigue comportant en son sein un ou plusieurs conflits qui doivent être résolus ou le développement de personnages plus ou moins complexes qui évoluent au fil du jeu. Puisque la fiction domine en popularité dans les médias audiovisuels, il n'est pas étonnant que le jeu vidéo en soit marqué et qu'il tente à son tour de se l'approprier d'une manière unique et spécifique.

Les arts visuels occidentaux, dont la peinture en première instance, ont construit au cours des siècles la structure de la représentation à l'intérieur d'un cadre. Même en ajoutant la reproduction technique du mouvement, le cinéma s'est essentiellement appuyé sur cette manière de représenter. De la même façon, le jeu vidéo a repris des arts visuels une grande partie des variantes représentationnelles proposées par les arts visuels. Des jeux de perspectives en 2D aux modélisations «sculpturales » en 3D, en passant par des images très abstraites ou extrêmement stylisées, le graphisme du jeu vidéo, construit en images de synthèse diverses (vectorielles, matricielles, 2D, 3D, etc.) a connu au cours de son développement une grande variété dans sa représentation, même à l'intérieur de quelques grandes formes générales. Pour mieux comprendre ce développement et les enjeux du jeu vidéo en tant que média audiovisuel, il est nécessaire de se pencher plus en détail sur la diversité de l'aspect visuel du jeu vidéo. ${ }^{8}$

\section{Les styles du jeu vidéo : de l'abstractionnisme au photoréalisme}

Une large portion des études vidéoludiques s'est intéressée aux structures interactives du jeu (principalement sa jouabilité) et aux questions narratives. ${ }^{9}$ Cet intérêt pour l'aspect ludologique (et narratologique) du jeu vidéo évacue le plus souvent toute question sur l'aspect «médiatique » du jeu vidéo. Pourtant, ce dernier est unanimement perçu comme un média audiovisuel. D'autant plus qu'une étude attentive de ses mécanismes ne peut que marquer la relation intime entre l'aspect visuel du jeu, la jouabilité, et l'expérience du joueur ; modalités qui s'influencent les unes sur les autres, et ce, du contexte de production au contexte de réception.

Lors du lancement d'un jeu, l'expérience du joueur commence d'abord et avant tout par un contact visuel avec celui-ci. Il doit le plus souvent « regarder» le jeu avant d'y jouer. Comme le mentionne Mark J.P. Wolf à propos de l'apprentissage singulier des joueurs afin d'acquérir une connaissance du vidéoludique, ces derniers ont dû, la plupart du temps, « lire » l'image pour comprendre le jeu (Wolf, 2001, p. 16). En ce sens, le joueur doit posséder ou acquérir une certaine littératie visuelle dans le contexte vidéoludique (Zagal, 2009 ; Leroux, 2016). Durant les années 1970 et le début des années 1980, les règles du jeu étaient déjà acquises en expérimentant visuellement avec le jeu. L'aspect visuel a toujours contribué au discernement des qualités esthétiques propres au jeu vidéo et à la compréhension de ses spécificités. 

principaux styles esthétiques du jeu vidéo, autant pour distinguer la manière dont ces styles permettent de réaliser des expériences esthétiques variées, que pour comprendre comment ils sont profondément liés à l'histoire visuelle du jeu vidéo. La notion de style a déjà été fréquemment utilisée et théorisée en histoire de l'art afin de caractériser les techniques dominantes en peinture, en architecture, en sculpture et autres, durant des périodes historiques différentes. Ce concept est aussi discuté en relation avec certains auteurs ou écoles particulières. Ainsi, l'étude d'un style individuel, bien que très fréquente en histoire de l'art et dans les études cinématographiques, est presque totalement absente des études du jeu vidéo. Cela s'explique entre autres par le manque d'approche auteuriale dans le jeu vidéo (qui fut pourtant essentielle pour légitimer le cinéma comme forme artistique), déterminé par plusieurs facteurs allant de ses modes de production à ceux de sa mise en marché. ${ }^{10}$

Alors qu'une caractérisation de styles auteurials dans le jeu vidéo peut encore être difficile à formuler, la tâche première est d'étudier les styles collectifs. En se concentrant sur ceux-ci, on peut remarquer dans l'histoire du jeu vidéo trois grandes tendances esthétiques, trois grands styles audiovisuels qui ont défini l'apparence des jeux. Aki Järvinen (2002) les a décrits par les termes suivants : l'abstractionnisme, le caricaturisme et le photoréalisme (Figure 1).

Figure 1

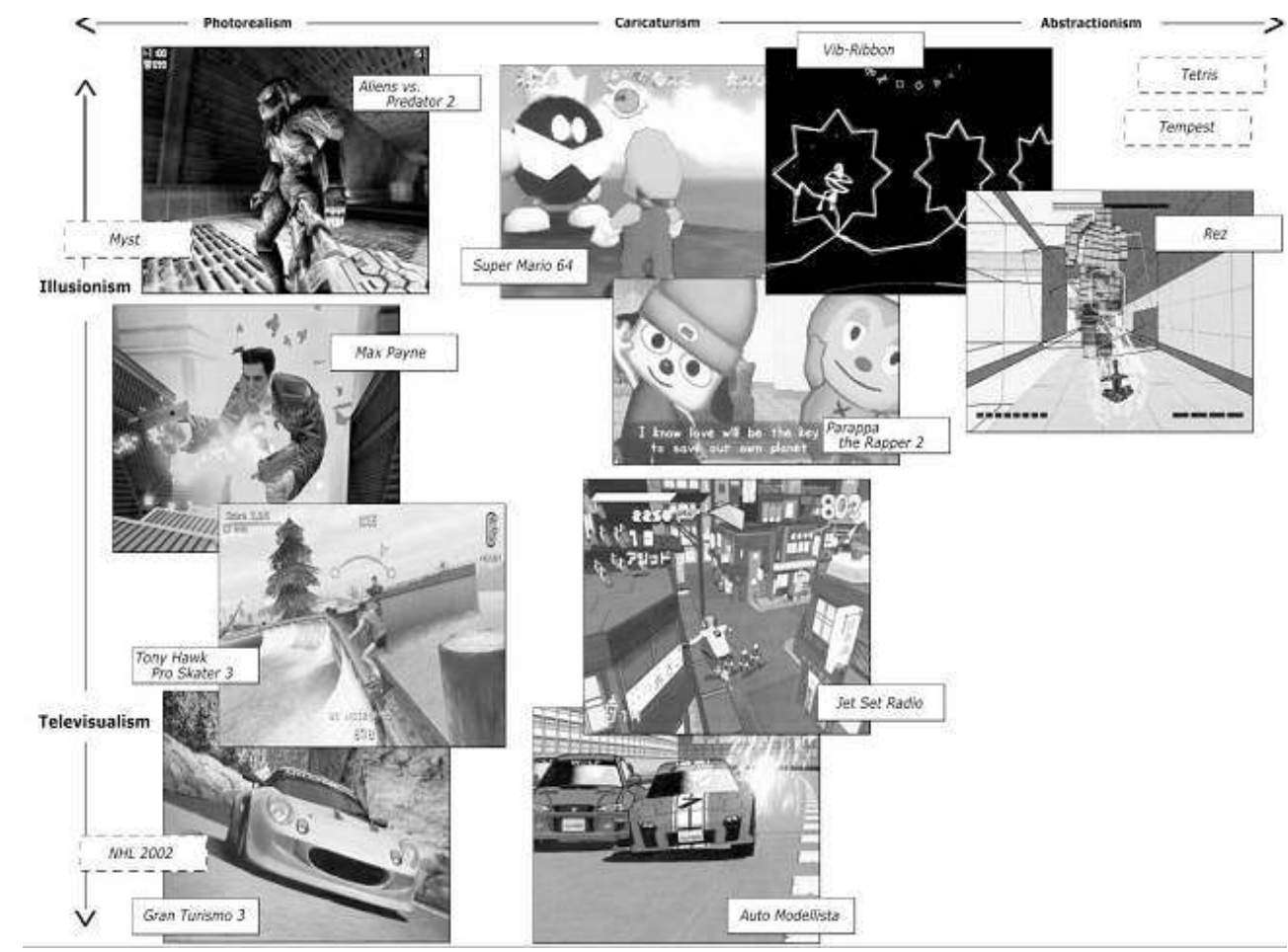

Le continuum des styles visuels de Aki Järvinen (2002)

L'intérêt de l'approche de Järvinen est d'avoir illustré les styles sur un continuum où les frontières entre les différents styles sont floues, et d'avoir montré que ce ne sont pas des catégories totalement distinctes, alors que certains jeux oscillent entre plus d'un style. Cette catégorisation peut d'ailleurs s'apparenter à celle établie par Scott McCloud dans Understanding Comics (1993), où il propose un tableau triangulaire dont les trois sommets 
sont le réalisme, l'iconicité et l'abstraction. Ce plan pictural s'adapte bien au degré d'iconicité des personnages vidéoludiques (Figure 2).

Figure 2

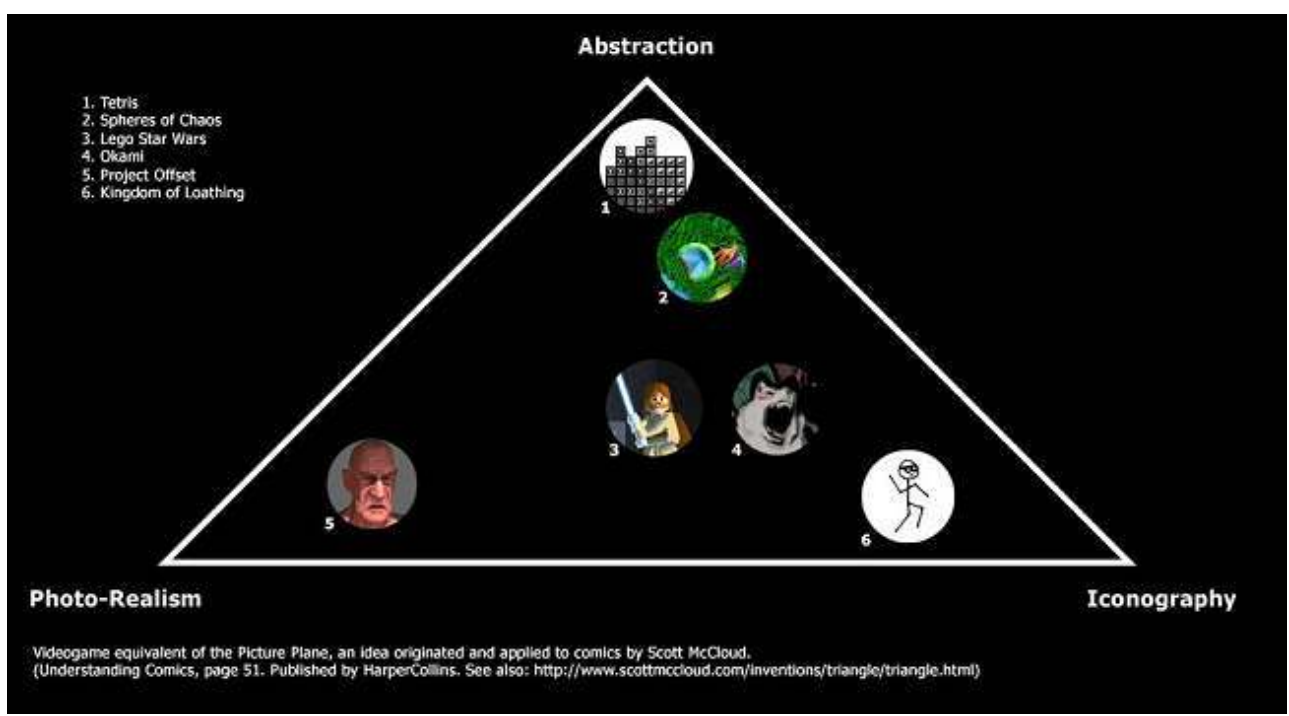

L'application du plan pictural de Scott McCloud au degré d'iconicité des personnages et objets dans le jeu vidéo (Hayward, 2005).

Selon Järvinen, l'abstractionnisme est le jeu des formes pures, où l'image est nonfigurative. C'est le style associé aux jeux de puzzle tels que Tetris (Alexeï Pajitnov, 1984). Par contre, pour Mark J.P. Wolf (2003), l'image abstraite n'est pas nécessairement non figurative. Elle est non représentative, c'est-à-dire qu'elle n'est pas à même de représenter des objets ou des personnages singuliers, sans qu'une interprétation en soit faite. Selon Wolf, l'abstraction est une simplification, une réduction d'un objet à ses formes et caractéristiques visuelles essentielles, plutôt qu'une tentative de reproduire cet objet en détail. Ce style caractérise les jeux vidéo des années 1970, où les limitations technologiques des images de synthèse ne permettaient pas la représentation réaliste des objets, des personnages et même des environnements. Depuis l'avènement du jeu vidéo indépendant, l'abstractionnisme a une forte valeur nostalgique alors que ce style est souvent repris par les concepteurs qui affectionnent les esthétiques "low-tech" afin d'offrir des expériences esthétiques très différentes de l'offre des jeux AAA (Camper, 2009).

Un style très populaire, surtout développé par l'industrie du jeu vidéo au Japon, est le caricaturisme. Tel que je le définis, les jeux caricaturaux sont des références directes ou indirectes aux représentations caricaturales de personnages et d'objets que l'on retrouve dans les bandes dessinées (manga inclus), les dessins animés (et anime) et les caricatures. Järvinen conçoit le caricaturisme comme la représentation d'un personnage ou d'un objet simplifié à ses traits les plus caractéristiques. Cependant, cette définition est semblable à celle donnée par Wolf de l'abstraction. Plutôt que la simplification, c'est surtout l'exagération qui est une des qualités propres au dessin animé et à l'animation, ${ }^{11}$ et qui définit son aspect visuel, surtout du côté des personnages. D'un point de vue de la conception de jeu, l'exagération est essentielle pour bien reconnaître et singulariser un personnage à l'écran. Le caricaturisme passe par la caractérisation des objets manipulables et une personnification des avatars, plutôt que par la simple figuration de 
ceux-ci. Comme le remarque Wolf (2003, p. 50), à l'époque de l'Atari 2600, seulement le jeu des couleurs permettait la distinction entre l'avatar, son outil, et l'environnement en arrière-plan. Par exemple, le jeu Golf (Atari, 1978) pour l'Atari 2600 ne permettait pas de singulariser les objets ou de personnifier l'avatar. Hors de son contexte (et sans connaître le titre du jeu), les objets graphiques étaient difficilement identifiables et facilement interchangeables (Figure 3).

Figure 3

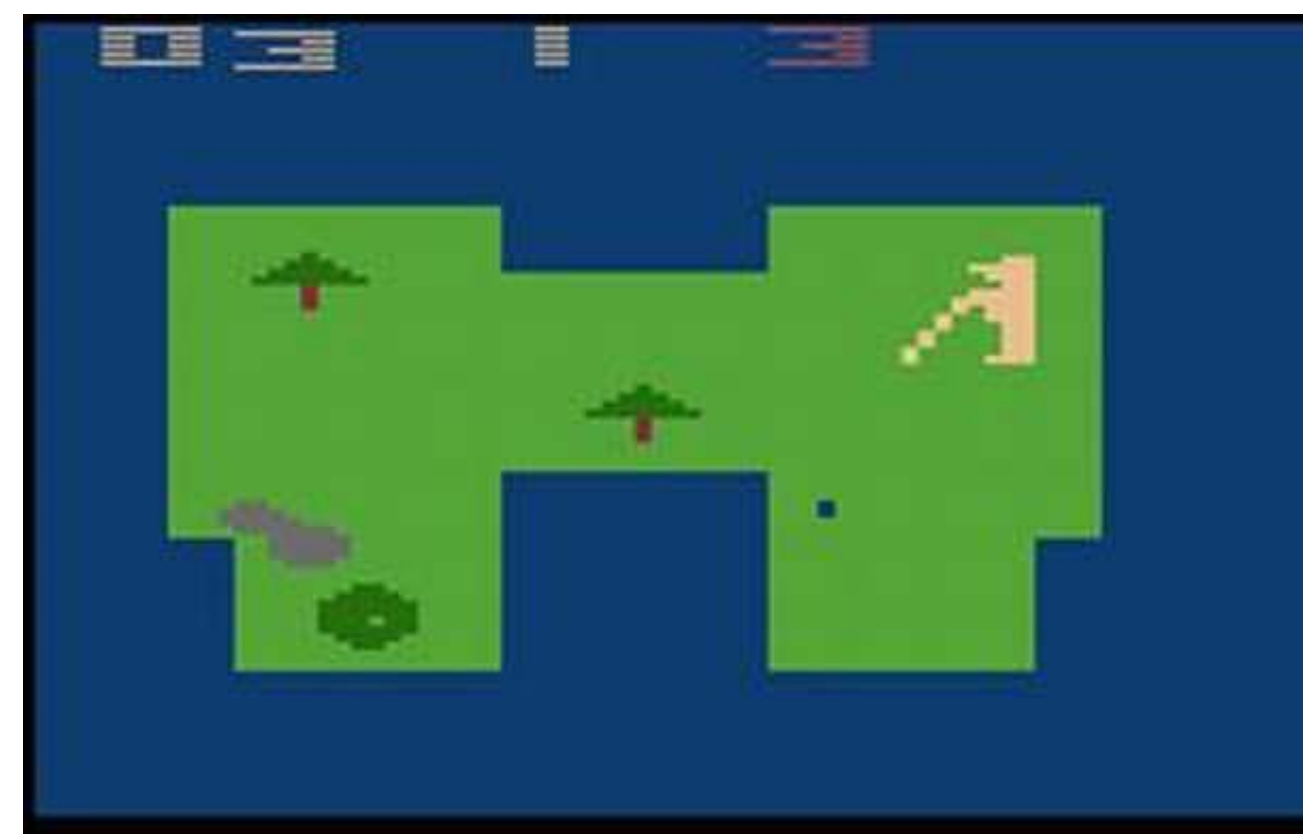

Le jeu Golf pour l'Atari 2600

D'ailleurs, l'environnement inaltérable, par son aspect fixe et statique, a toujours bénéficié des graphismes les plus détaillés, contrairement aux personnages qui furent pour une longue période tirés à grands traits. Par exemple, Altered Beast (Sega, 1988), jeu d'arcade qui deviendra l'année suivante le jeu de lancement de la Mega Drive en Europe et de la Genesis en Amérique du Nord, est un jeu à défilement horizontal (side-scrolling) qui illustre une technique appelée le « défilement différentiel » (parallax scrolling) afin de donner une impression de profondeur à l'environnement à travers des portions de décors ou des plans défilant à vitesse légèrement variable. Cette technique, adaptée d'un procédé qui fut développé pour le dessin animé sur celluloïd, permit de donner une "perspective de mouvement ", et par conséquent produisait un effet évident de multiplanarité. L'année suivante, le jeu Shadow of the Beast (Psygnosis, 1989) sur Amiga géra jusqu'à treize plans à défilement différentiel simultanément (Figures 4 A-B). 
Figure 4A

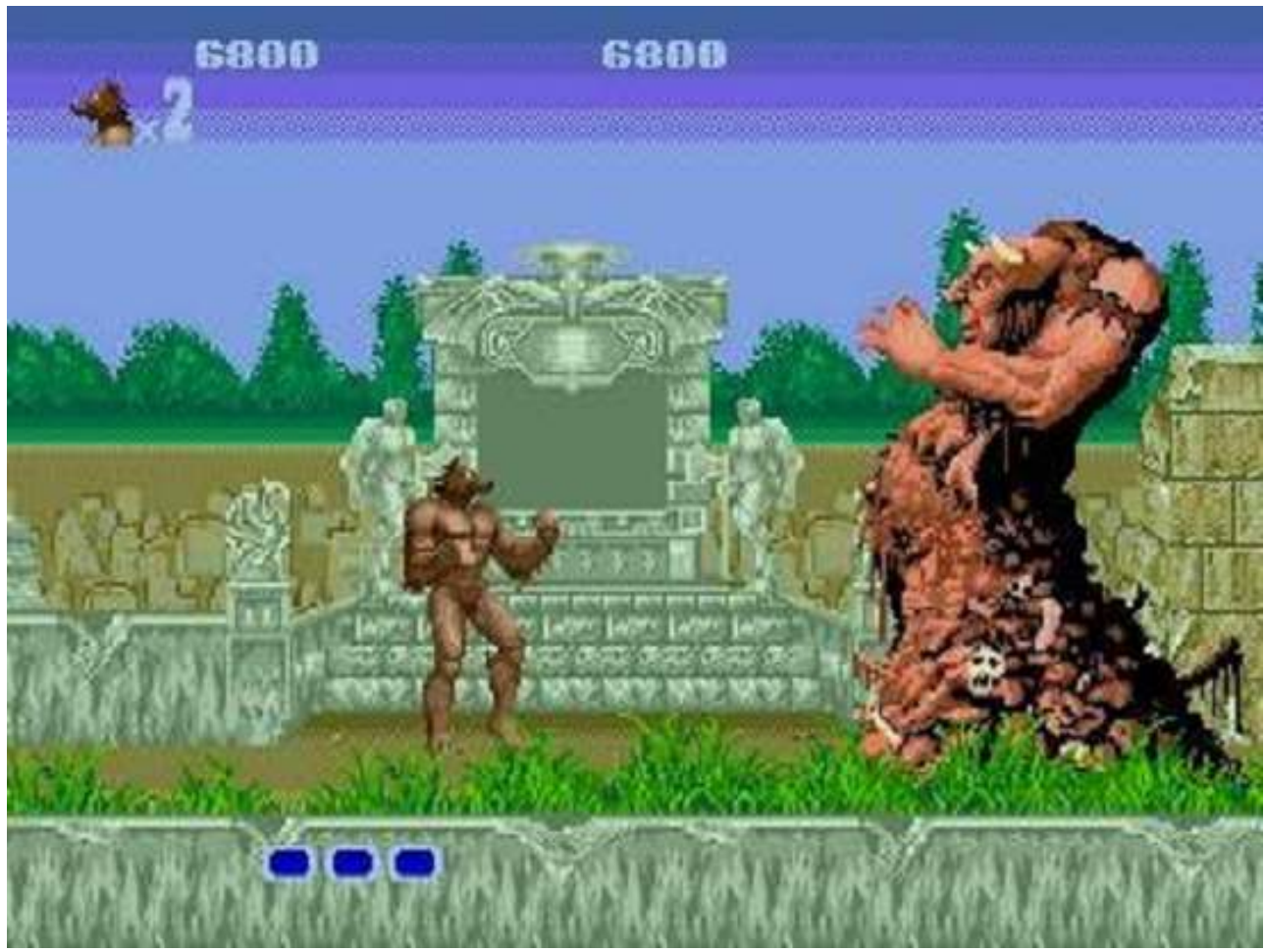

Altered Beast. Exemple de defilement différentiel

Figure 4B

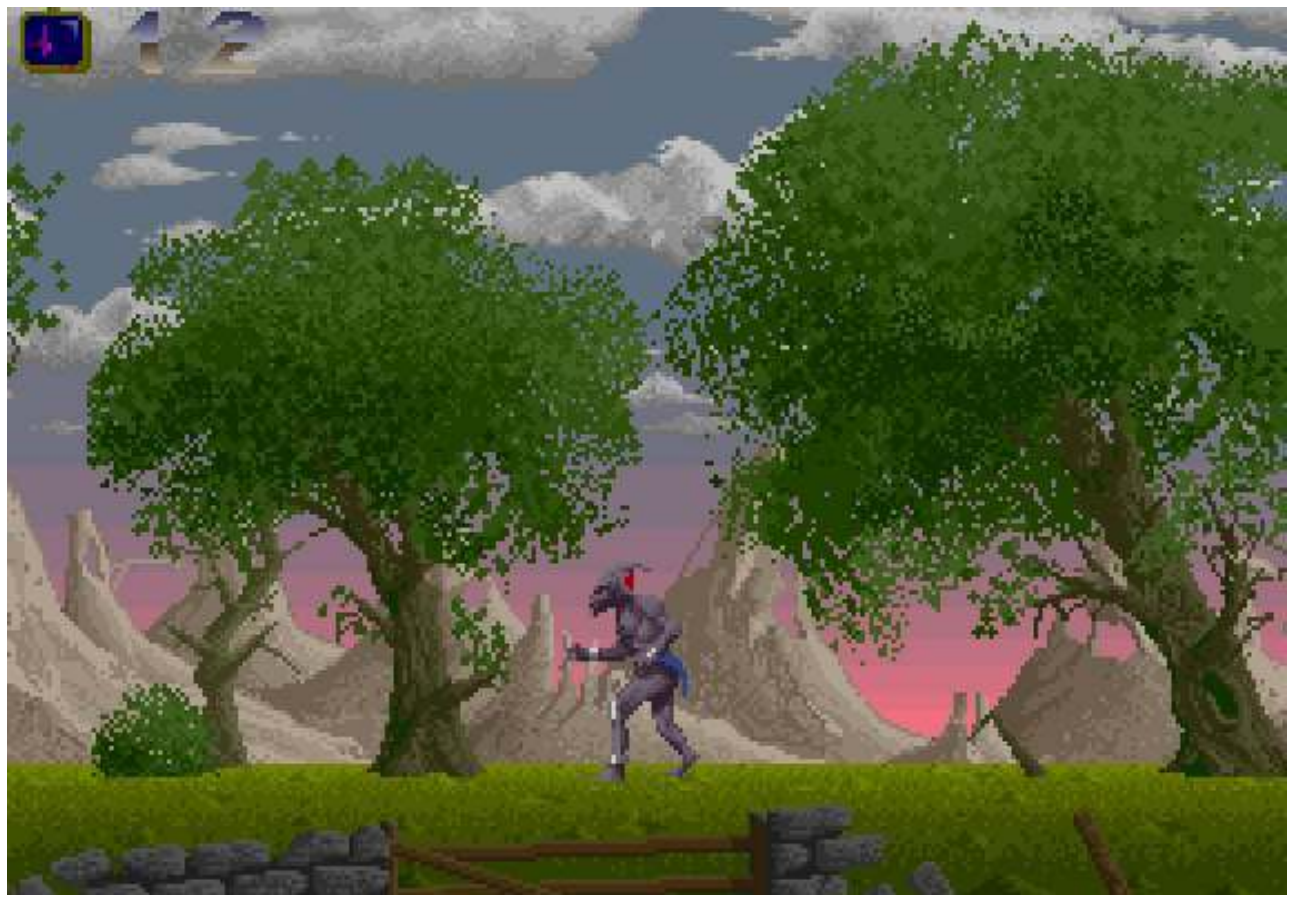

Shadow of the Beast. Autre exemple de défilement différentiel

24 Puisque le défilement différentiel vise à susciter un effet de profondeur à l'aide de multiples plans 2D, il a permis de proposer un effet marqué de (photo)réalisme dans les 
jeux vidéo. Il a souvent été souligné que le photoréalisme était caractérisé par la remédiatisation des conventions du réalisme cinématographique (Bolter et Grusin, 1999 ; Poole, 2000 ; Manovich, 2001; King et Krzywinska, 2002 ; Lister et alii, 2003), plutôt que par une simple «reproduction» de la réalité. Quoi qu'il en soit, il est problématique de considérer le photoréalisme comme l'aboutissement d'une évolution téléologique des graphismes du jeu vidéo vers une illusion toujours plus réussie. Bien que l'intention réaliste et illusioniste est une tendance dominante dans l'histoire du graphisme vidéoludique, comme ce fut le cas d'ailleurs durant la majeure partie de l'Histoire de l'Art (Gombrich, 2002 ; Grau, 2003), elle demeure tout de même une option (esthétique) parmi d'autres.

\section{Les styles à travers l'histoire du jeu vidéo}

Les styles ont bien entendu évolué au cours de l'histoire du jeu vidéo, l'appellation "photoréalisme » ne faisant son apparition qu'à l'arrivée de l'image de synthèse 3D texturée en temps réel, tandis que l'abstractionnisme (appellation utilisée à rebours) et le caricaturisme furent présents depuis les débuts (ou du moins très tôt dans le cas du caricaturisme). En regardant l'histoire des styles audiovisuels dans les jeux vidéo, il est évident que certaines époques ont privilégié certains styles. Cela n'a toutefois pas empêché la présence de styles alternatifs qui proposaient souvent des expériences fort différentes et originales.

Pour mieux comprendre cette évolution, j'ai illustré les tendances stylistiques à cinq époques différentes, correspondant aux années 1978, 1988, 1998, 2008, et 2016 (cf. Annexe). En 1978, c'est le début de la grande popularité des jeux d'arcade, avec principalement le boom Space Invaders (Taito, 1978). Dans ce jeu, et les autres de l'époque (Figures 5 A-B-C-D), les objets ont une apparence abstraite puisqu'ils se limitent à des formes géométriques simples. Quant aux personnages, ils ne possèdent pas de traits individuels ni de personnalité, comme on le verra quelques années plus tard avec entre autres Pac-Man (Namco, 1980).

Toutefois, à défaut d'être réalistes, quelques tentatives de représentation figurative étaient amorcées. Le jeu d'arcade Frogs (Gremlin, 1978) fut un des premiers jeux à représenter un animal, en l'occurrence une grenouille (l'avatar) et un insecte, que le joueur devait attraper. Puisque la technologie graphique était limitée, la stratégie de l'époque fut de compenser cette lacune en créant des cabinets d'arcade décorés par les personnages et les environnements, bref un contexte narratif, qui devaient figurer à l'écran. Les concepteurs de Frogs ont poussé cette idée plus loin en créant un avant-plan dessiné sur l'écran de la cabine pour représenter l'environnement, à la façon des jeux de la première console de salon, l'Odyssey de Magnavox (1972). 
Figure 5A

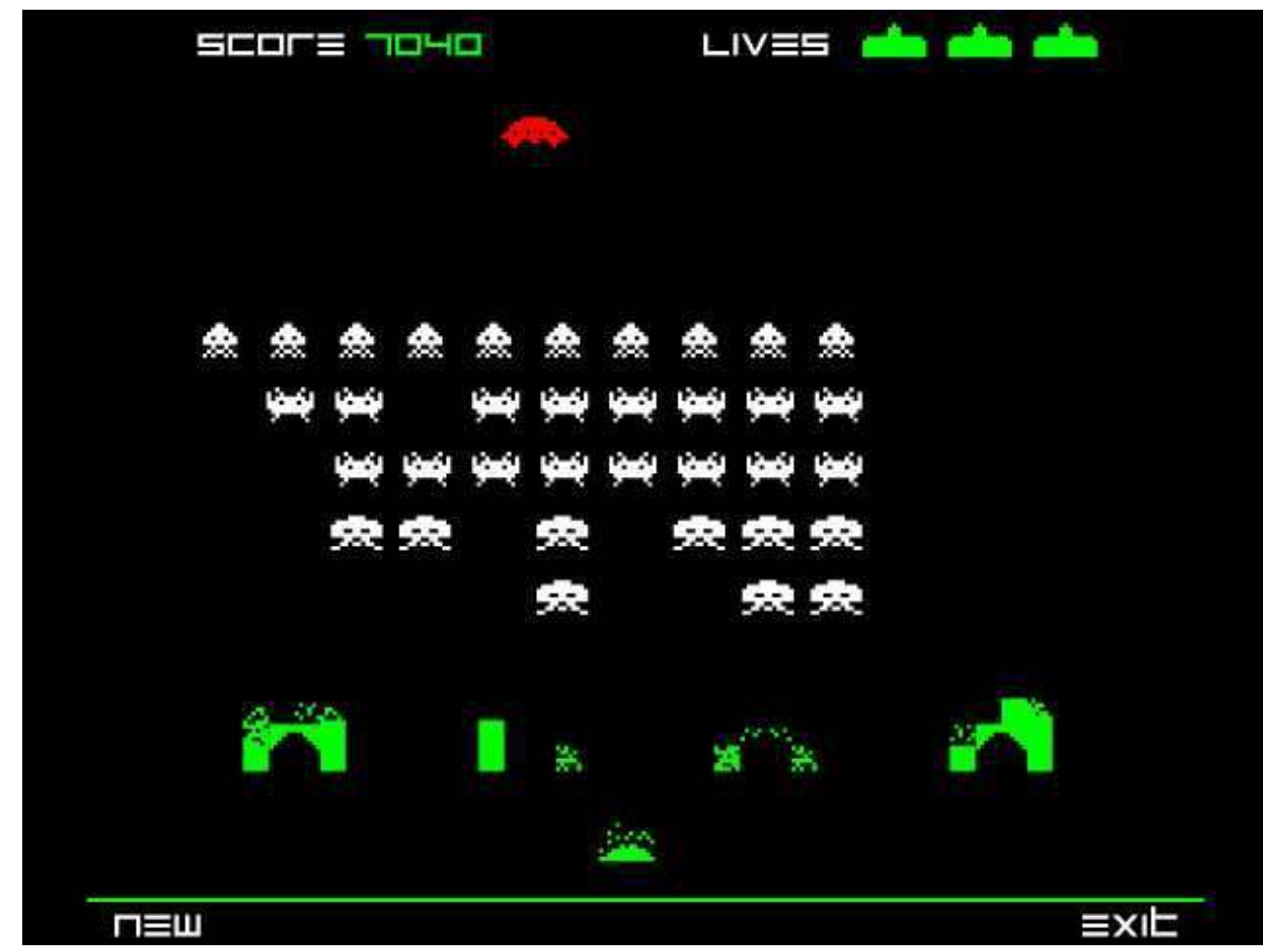

Space Invaders

Figure 5B

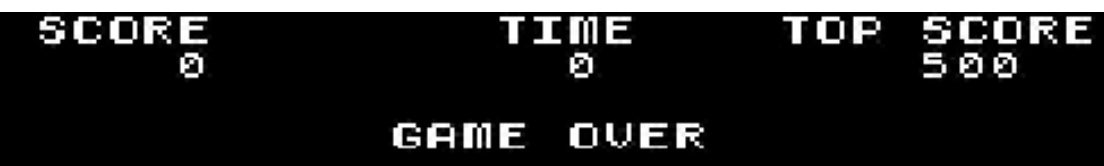

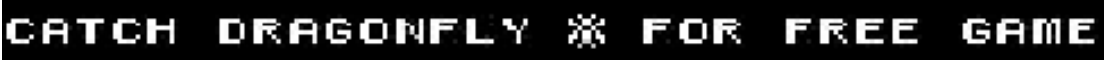

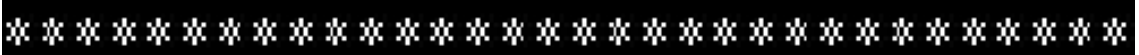

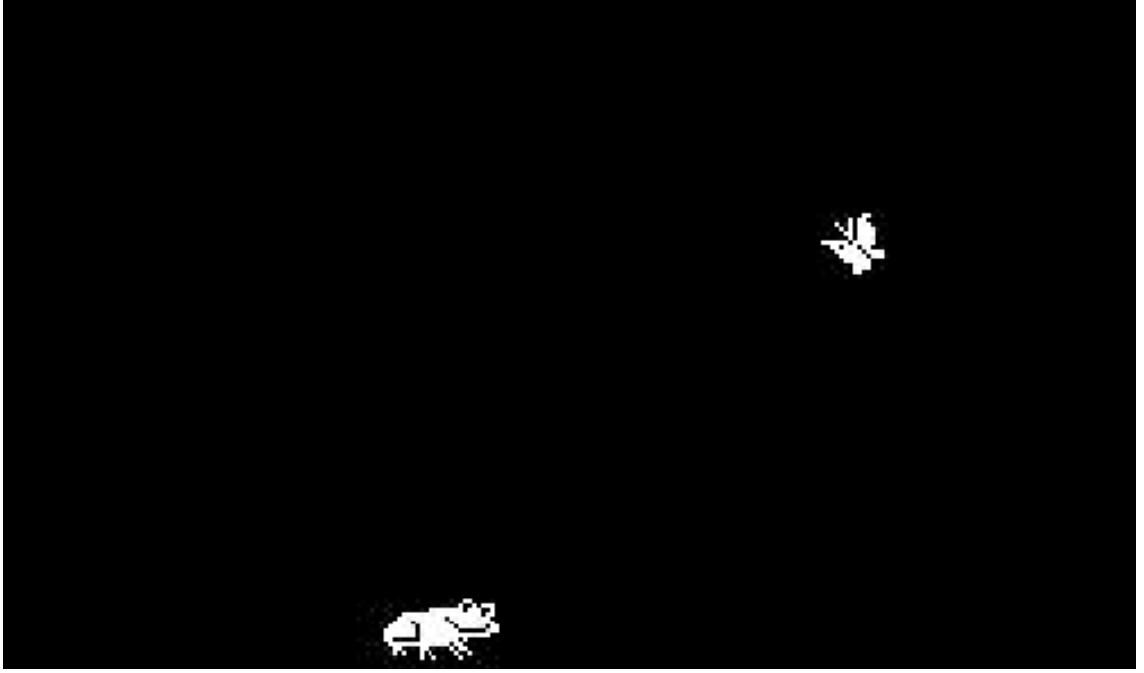

Frogs (l'écran de jeu) 
Figure 5C

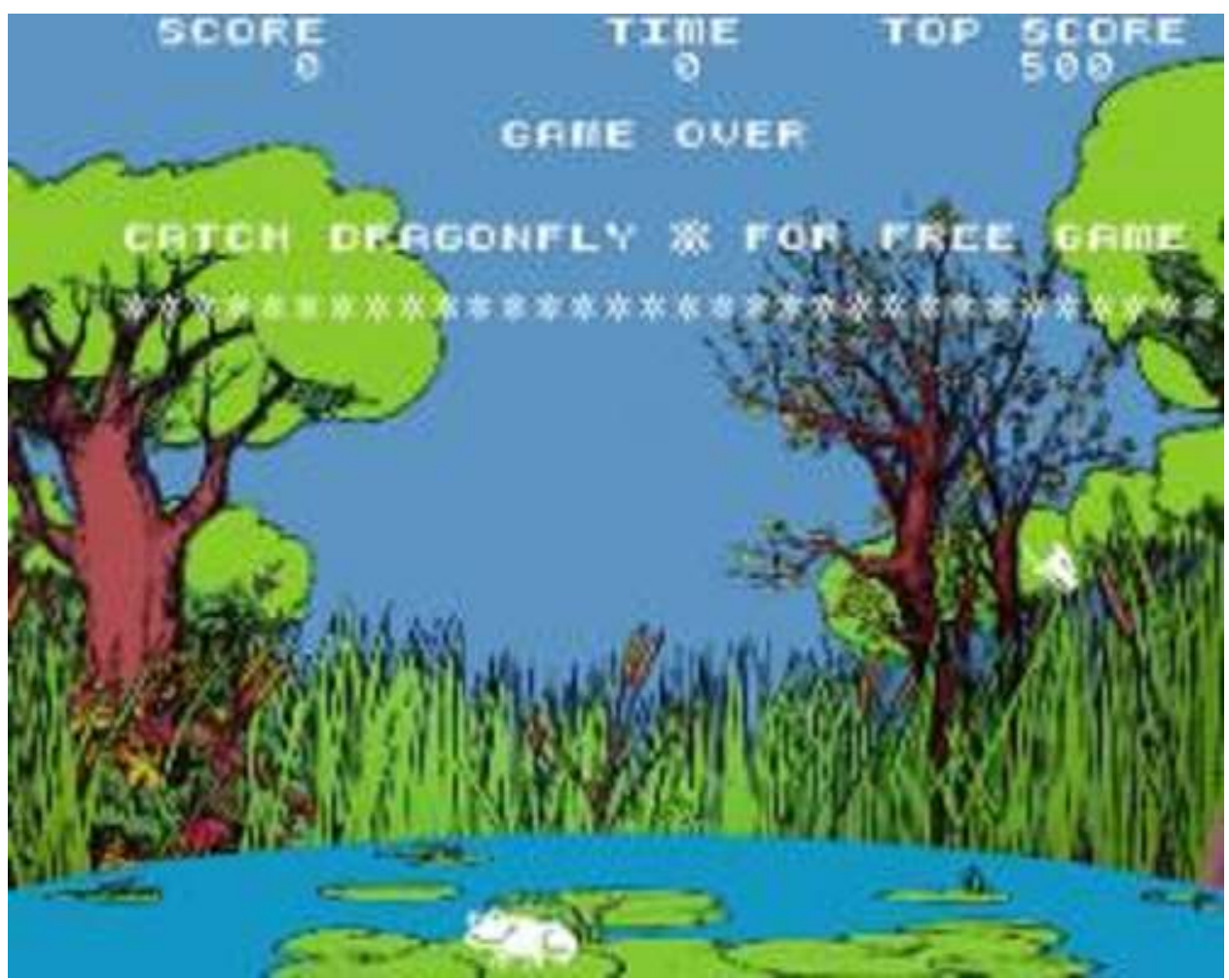

Frogs (la superposition de l'arrière-plan)

\section{Figure 5D}

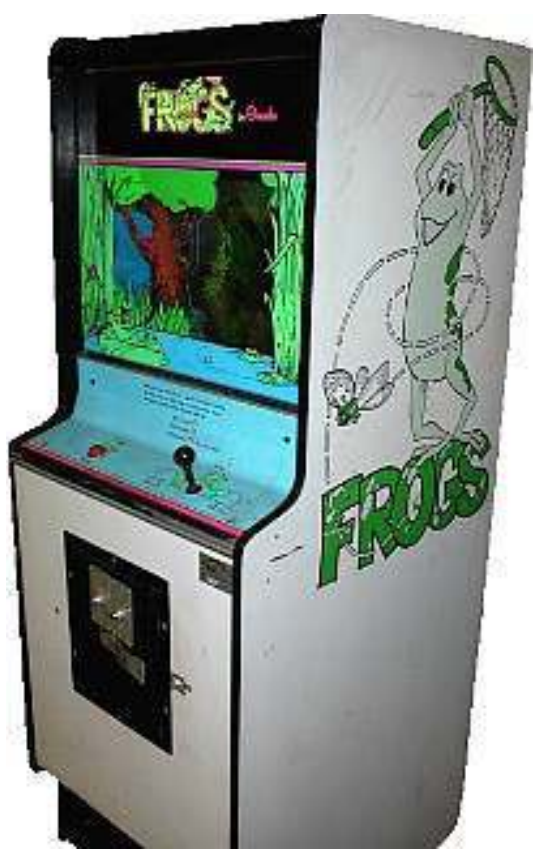

Frogs (la borne d'arcade).

28 À mesure que les technologies graphiques se sont développées, les graphismes sont devenus de plus en plus figuratifs, sans nécessairement être réalistes. En 1988, c'est 
l'apogée des consoles 8-bit alors que le style caricatural domine de façon convaincante. Dans les jeux d'arcade, comme Ghouls' $n$ Ghosts (Capcom, 1988) ou les jeux sur console, peu importe leurs genres, que ce soit un jeu de course comme R.C. Pro-Am (Nintendo, 1988) ou un jeu de rôle tel que Final Fantasy II (Square, 1988), les personnages et les objets manipulables (comme les voitures) sont représentés de façon caricaturale (Figures 6 A-BC). Ce style, similaire aux dessins animés, souligne d'autant plus que les jeux de l'époque s'adressaient essentiellement à un jeune public.

Figure $6 \mathrm{~A}$

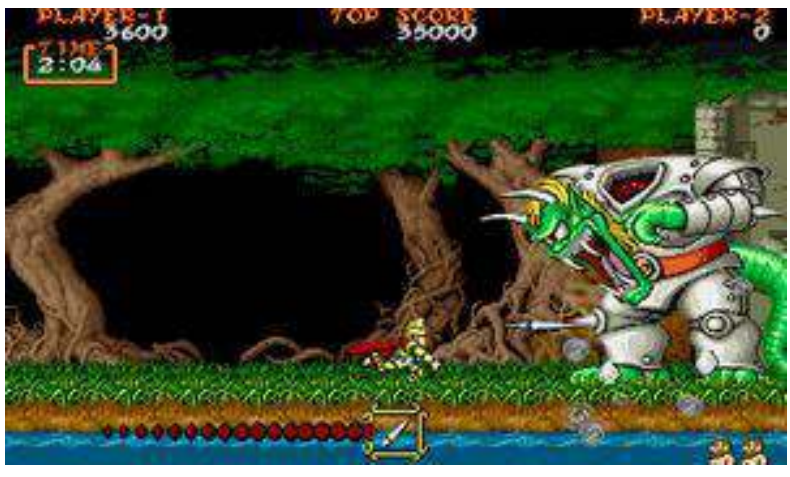

Ghouls'n Ghosts

Figure 6B

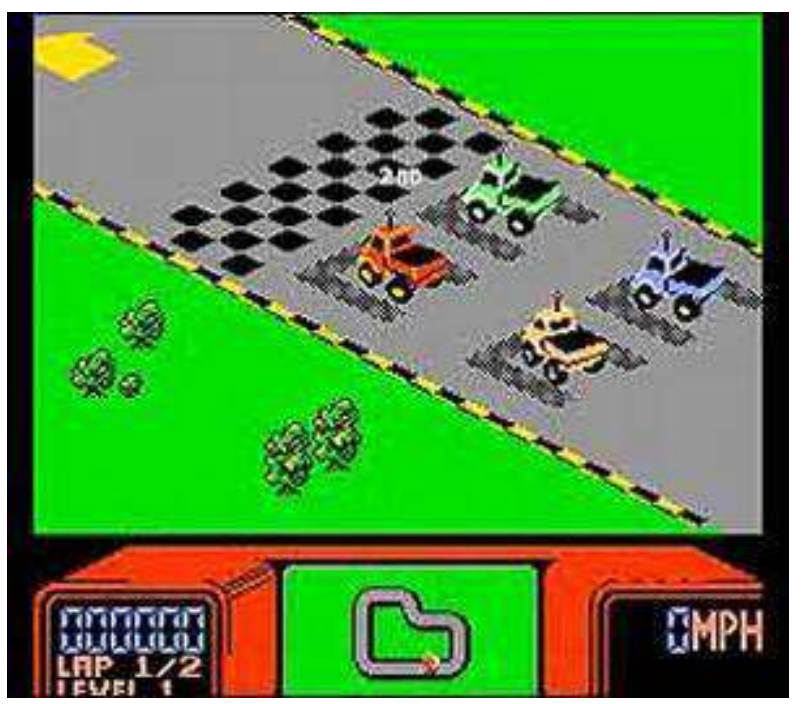

R.C. Pro-Am 
Figure 6C

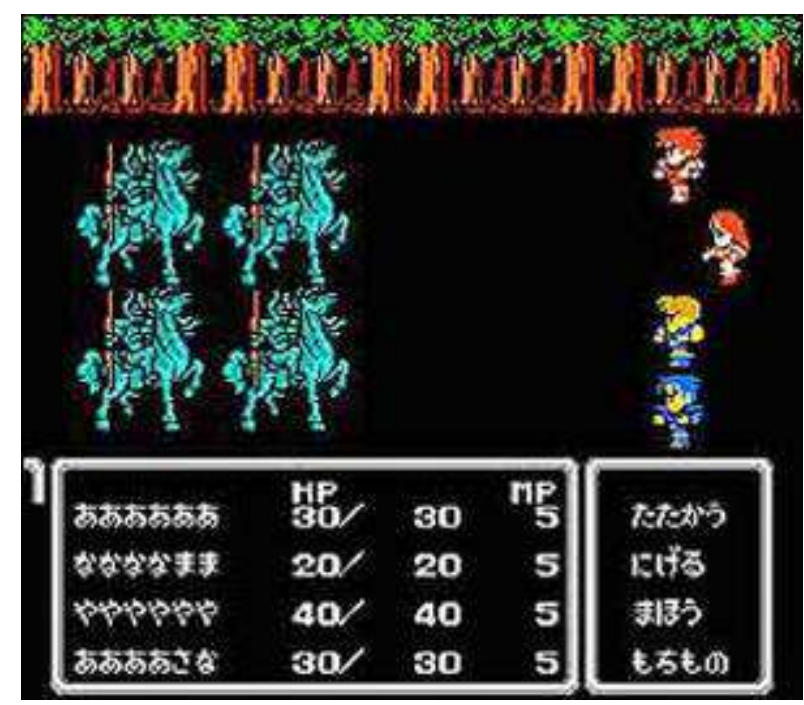

Final Fantasy II

Durant cette période, les concepteurs se mirent à utiliser diverses stratégies visuelles afin de donner du réalisme aux images vidéoludiques. Par exemple, l'arrivée de la 3D non texturée développée dans l'animation par ordinateur et les effets visuels au cinéma dès le début des années $1980^{12}$ marqua un pas conséquent vers le photoréalisme. Les jeux de simulation, tels que F/A-18 Interceptor (Intellisoft, 1988), poussèrent la technologie dans ce sens, puisque le plaisir du joueur face à ce genre découlait directement de la possibilité d'une expérience la plus mimétique possible (Figures 7 A-B).

Figure 7A

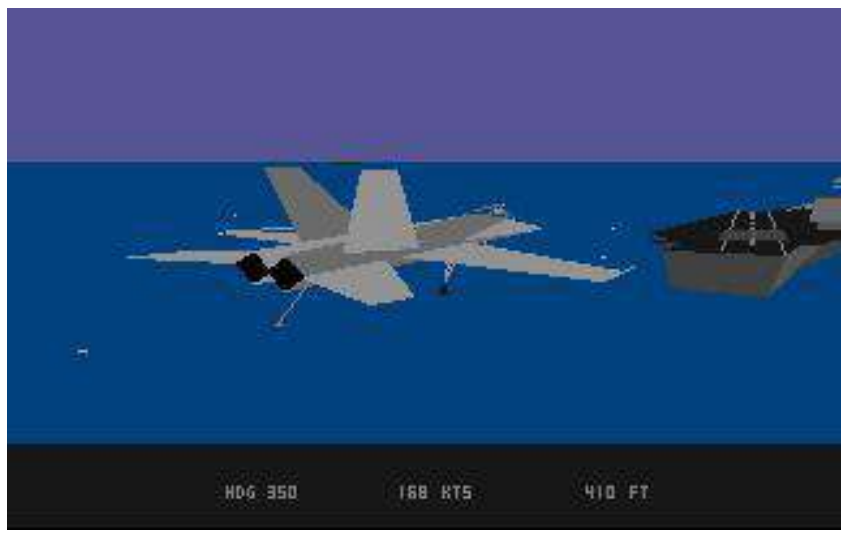

F/A-18 Interceptor. 
Figure 7B

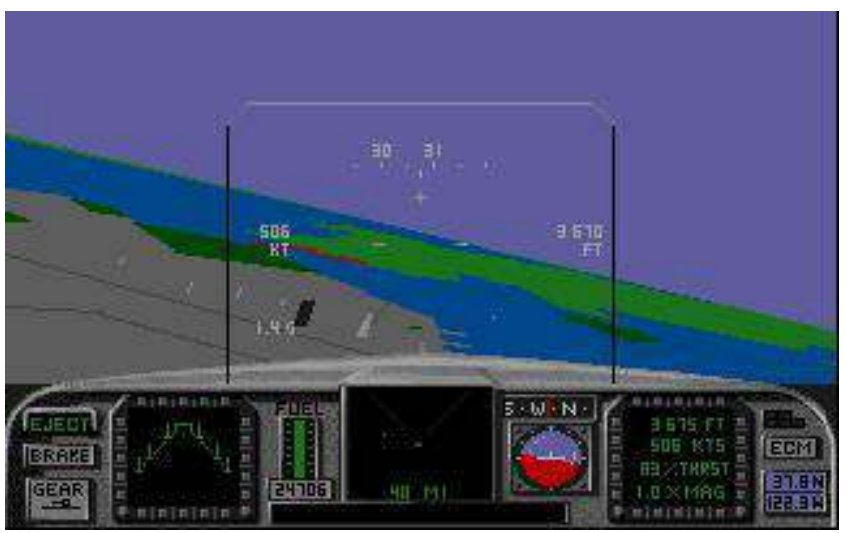

F/A-18 Interceptor.

30 À la fin des années 1980, l'abstraction est encore présente, mais beaucoup moins fréquente. On assiste à des expérimentations de jeux en 3D comme Virus (Frontier Developments, 1988) ou aux jeux de simulation sur les premiers ordinateurs personnels, comme la première itération de la très populaire série John Madden Football (1988-) sur le Commodore 64, où les limitations du matériel informatique restreignent les graphismes au stade de l'abstraction (Figures 8 A-B). Cette série, tout comme celle de Microsoft Flight Simulator, deviendra d'ailleurs l'un des baromètres majeurs de l'évolution technologique de l'image de synthèse 3D (Arsenault et Côté, 2013).

\section{Figure 8A}

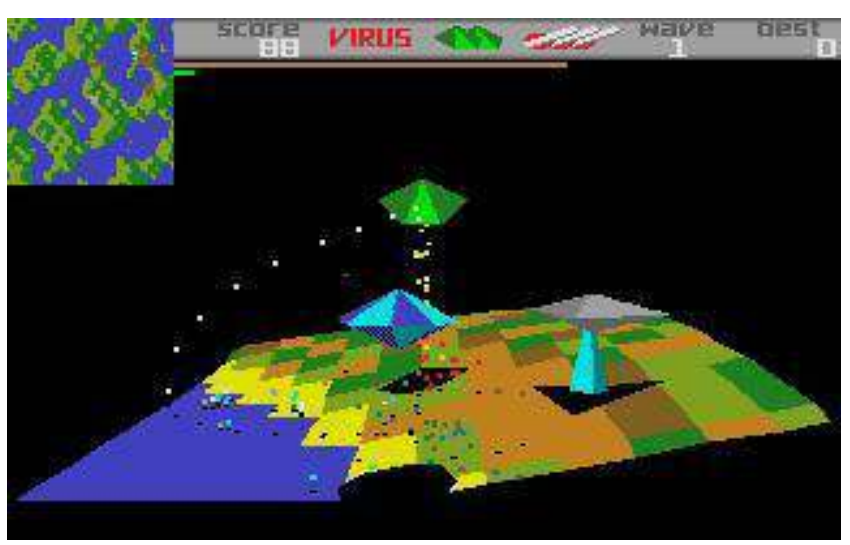

Virus 
Figure 8B

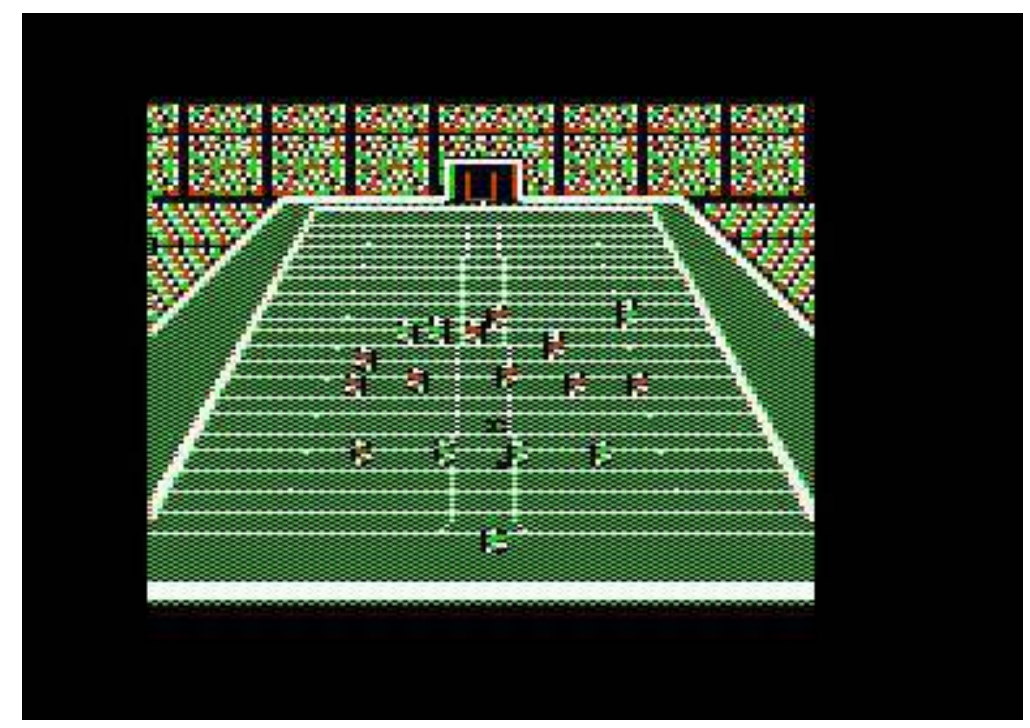

John Madden Football

31 Dix années plus tard, en 1998, c'est l'apogée de l'engouement pour le photoréalisme et des promesses de l'image de synthèse texturée. Au début des années 1990, l'arrivée des images de synthèse en 3D texturée dans le cinéma d'animation et d'effets visuels permet aux jeux vidéo de profiter de ces technologies. C'est le cas principalement de l'ordinateur personnel (PC) qui supporte désormais les cartes graphiques accélératrices 3D et qui popularise le nouveau support CD-ROM qui profite d'un plus grand espace de stockage. Malgré la popularité qu'entraîne le photoréalisme, principalement dans les magazines spécialisés, certains jeux demeurent caricaturaux, principalement ceux de la console Nintendo 64, alors que Nintendo refuse le passage technologique de la cartouche au CDROM. Le caricaturisme se remarque également dans les derniers jeux d'aventure sur PC de la compagnie LucasArts, tels que Grim Fandango (LucasArts, 1998). Ce jeu, profitant d'un style marginal pour l'époque, est remarqué pour ses qualités artistiques, plutôt que technologiques (Figures $9 \mathrm{~A}-\mathrm{B}$ ).

Figure 9A

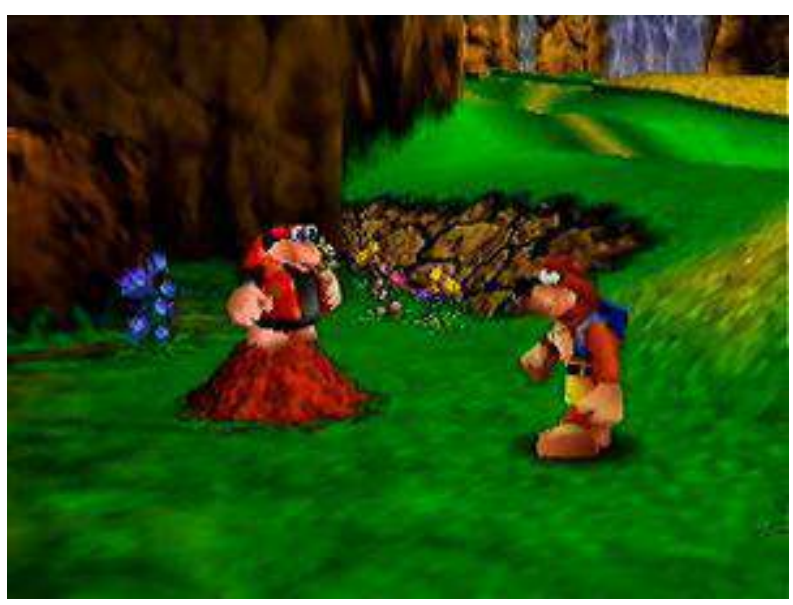

Banjo-Kazooie 
Figure 9B

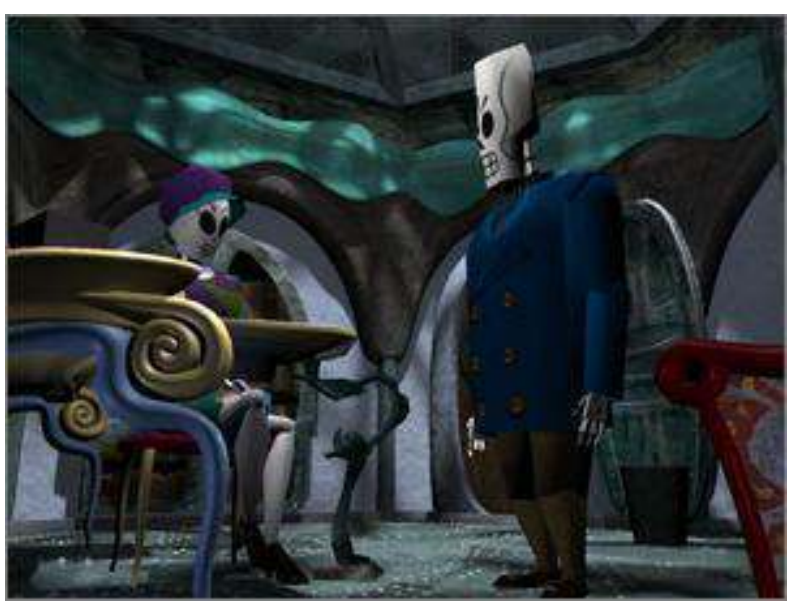

Grim Fandango

32 Toutefois, la majorité des jeux de l'année 1998 s'insère dans la catégorie photoréaliste (qui paraît bien primitif aujourd'hui, alors qu'il est nécessaire de se remettre dans le contexte de l'époque). Ce style se caractérise entre autres par le «télévisualisme » des jeux de sports tels que le jeu de planche à neige $1080^{\circ}$ Snowboarding (Nintendo, 1998), où les positions de caméra rappellent le reportage télévisé. Le photoréalisme est également utile pour créer des univers fictifs convaincants comme dans le jeu d'horreur Resident Evil 2 (Capcom, 1998), alors que l'atmosphère détaillée de ce jeu met en évidence les performances impressionnantes de la console PlayStation de Sony. Les jeux dont la thématique repose sur la science-fiction jouissent aussi de cet avantage technologique alors que le jeu Half-Life (Valve, 1998) marque un précédent en matière de photoréalisme (Figures $10 \mathrm{~A}-\mathrm{B}-\mathrm{C}-\mathrm{D})$.

Figure 10A

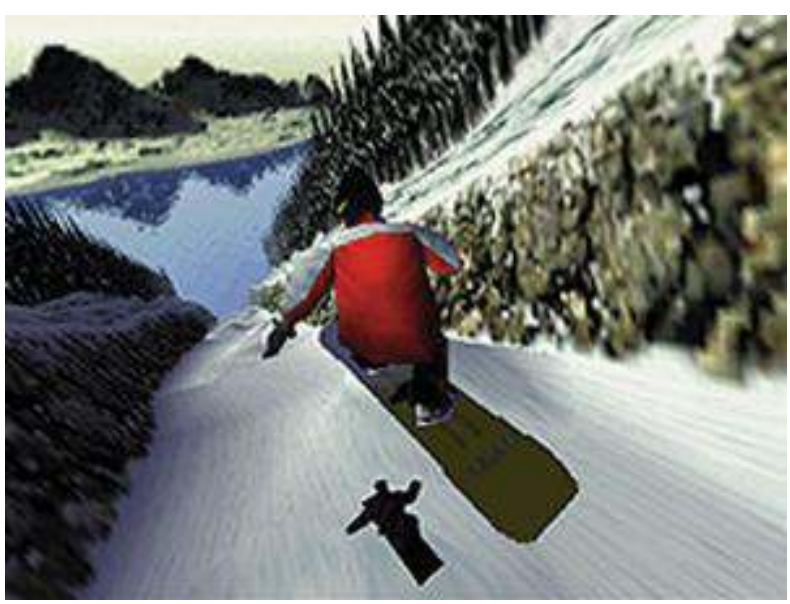

$1080^{\circ}$ Snowboarding 
Figure 10B

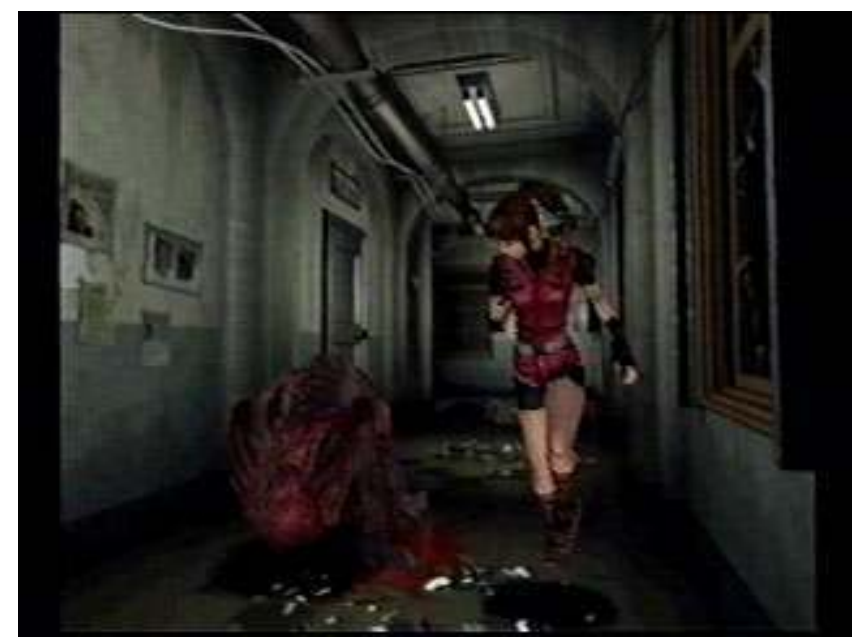

Resident Evil 2

Figure 10C

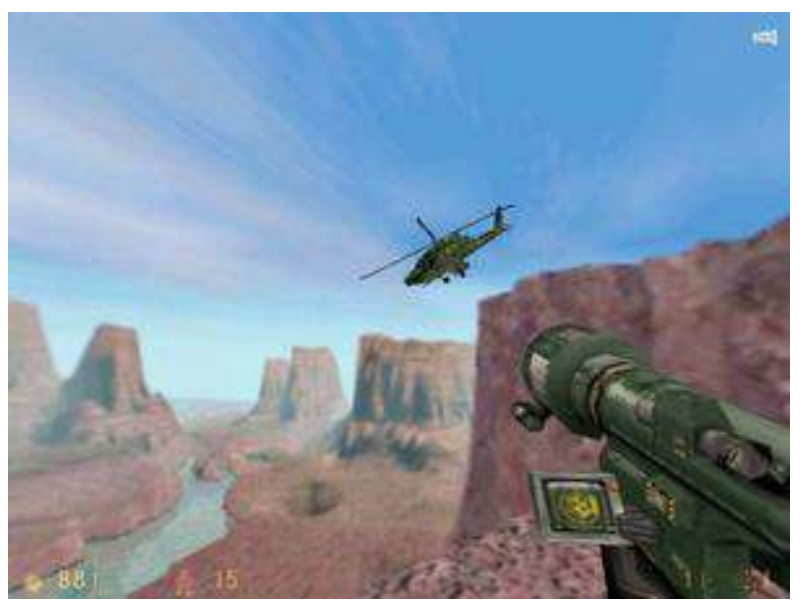

Half-Life

Figure 10D

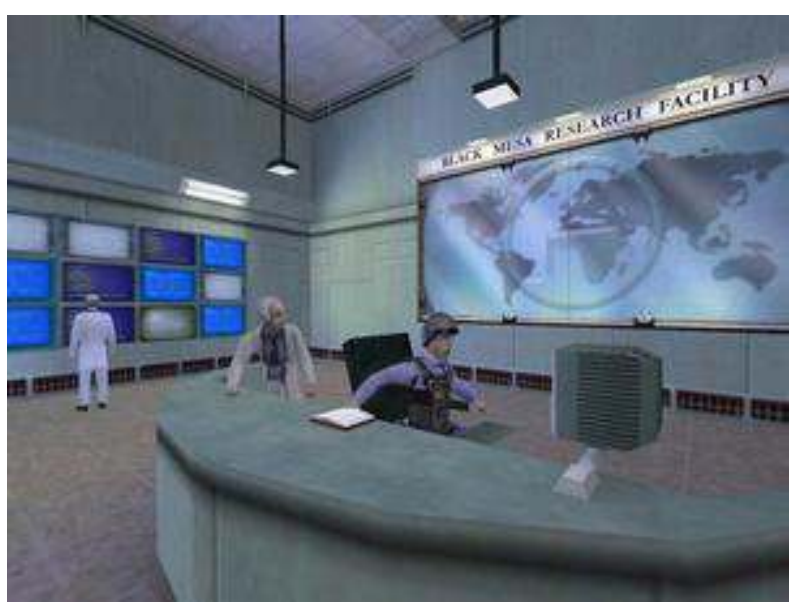

Half-life 
Par la suite, il y eut au début des années 2000, comme l'a supposé Järvinen (2002), une saturation des jeux photoréalistes (du moins en Amérique du Nord), alors que les concepteurs commencèrent à s'ouvrir à des alternatives esthétiques, ou plutôt à revisiter les styles caricaturaux et abstraits. En 2008, si le photoréalisme dominait toujours, un attachement nostalgique s'est développé pour les premières formes vidéoludiques, que ce soit par le remake du jeu abstrait Rez (Q Entertainement, 2001) (maintenant en haute définition) sur la console Xbox 360 (Rez HD [Microsoft, 2008]), ou par l'intérêt soutenu pour les jeux de puzzle simples, tels que $\mathrm{N}^{+}$(Metanet Software, 2008), disponible sur les consoles portables Nintendo DS et PSP. Ce jeu récupère le style graphique des titres de la console Atari 2600 (commercialisée en 1977) et des autres consoles domestiques de la fin des années 1970 et du début des années 1980. Finalement, l'abstractionnisme est le style privilégié pour certains jeux expérimentaux ou indépendants comme le jeu ambiant flow (thatgamecompany, 2008), adapté en téléchargement sur la PS3 et la PSP (Figures 11 A-BC-D).

Figure 11A

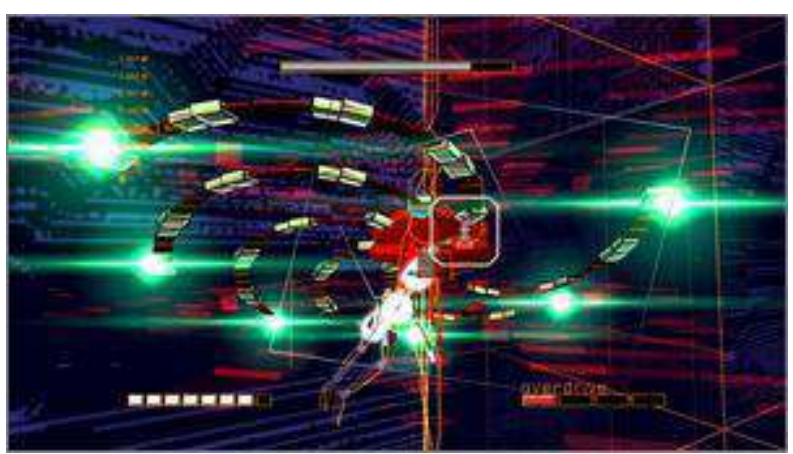

REZ HD 
Figure 11B

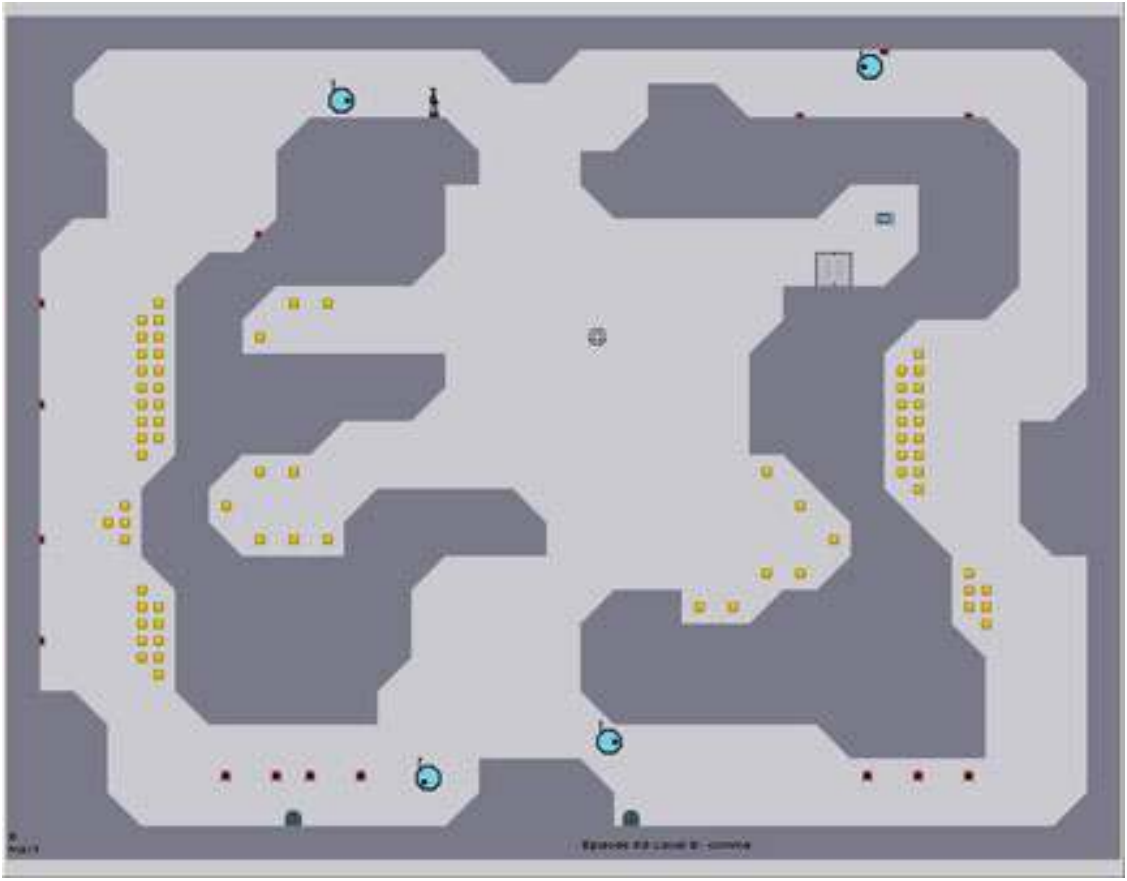

$\mathbf{N +}$

Figure 11C

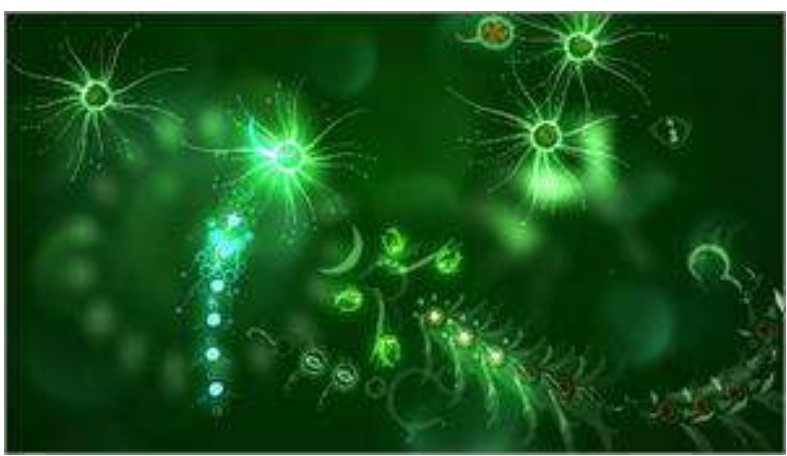

FLOW

Figure 11D

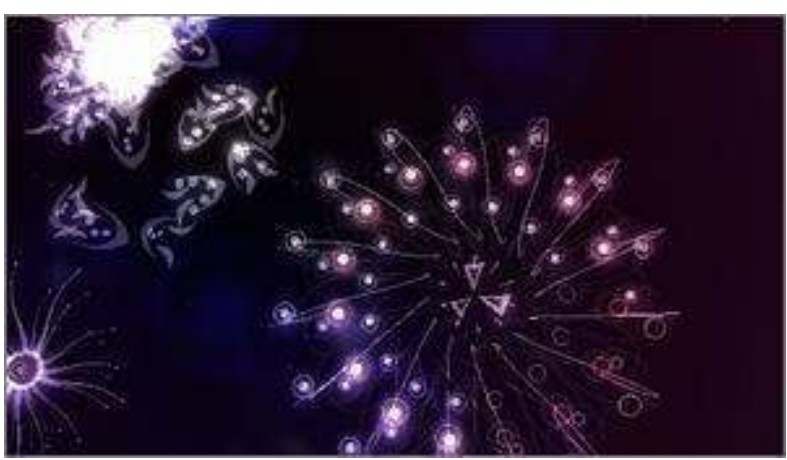

FLOW 
34

me on a pu le remarquer, le développement technologique a tout de même fortemen marqué l'évolution des styles visuels du jeu vidéo durant les trois premières décennies. Maintenant que la croissance de la technologie 3D n'est plus exponentielle, la diversité des styles se fait davantage sentir, avec même un retour en force du caricaturisme depuis quelques années grâce à l'essor du jeu vidéo indépendant et d'une certaine forme de nostalgie envers les jeux d'antan. Ainsi, cela n'est pas uniquement dû aux aspects technologiques, alors que plusieurs autres facteurs influencent le contenu de ce que l'on peut voir à l'écran.

\section{Facteurs de contribution aux styles}

À travers cette catégorisation, il est possible de distinguer divers facteurs qui conditionnent les styles audiovisuels des jeux. Comme mentionné, il n'y a pas que le facteur technologique, soutenu par la tendance téléologique d'un photoréalisme toujours plus achevé, bien que celui-ci ait longtemps joué un rôle majeur. Autrement, le genre vidéoludique est également un aspect déterminant pour le style d'un jeu. Par exemple, le jeu de rôle sur console, provenant essentiellement du Japon durant les années 1980 et 1990, est presque toujours caricatural étant donné qu'il s'est développé à la fois dans un contexte collaboratif entre les principales industries culturelles (manga, anime et jeu vidéo; Aoyama et Izushi, 2006) que sous le ciblage d'un jeune public friand de manga et d' anime (Kohler, 2005). Les jeux à thématique guerrière et autres jeux de simulation ont le plus souvent privilégié le photoréalisme (avec quelques exceptions telles que le caricaturisme de Team Fortress 2 [2007]), tandis que les jeux de puzzle continuent à privilégier généralement l'abstraction. L'abstraction est associée aujourd'hui aux jeux artistiques et expérimentaux, et à l'esthétique «low tech» de plusieurs développeurs indépendants.

Plusieurs facteurs socioculturels et économiques influencent les styles privilégiés selon l'époque, à commencer par le ciblage du public chez les distributeurs, et les attentes des joueurs (la conviction générale étant que les enfants préfèrent le caricaturisme, et les adultes, le photoréalisme). Il est évident que la génération des années 1980 n'est pas la même que celle d'aujourd'hui, surtout en ce qui a trait aux attentes et aux types d'expériences voulues. La génération des années 1970 et 1980 était celle de la découverte du jeu vidéo, comme le spectateur de cinéma du début du $\mathrm{XX}^{\mathrm{e}}$ siècle, alors elle acceptait plus facilement les graphismes abstraits ou peu figuratifs de l'époque. Selon Wolf (2003), les jeux abstraits sont d'ailleurs remarquables par leur adéquation entre ce que l'on voit à l'écran et ce que l'on doit faire. La jouabilité de Tetris repose entièrement sur le jeu des formes (visuelles). Le fait qu'un jeu comme Death Race (Exidy, 1976) provoqua une controverse due à sa violence graphique « trop explicite » (au goût des parents) démontre bien que la question du réalisme des images doit toujours être contextualisée.

Ainsi, les types de joueurs selon les époques déterminent également les préférences pour certains styles. Jouer en solo (single player), en co-op, ou en ligne n'entraîne pas les mêmes attentes ; de même pour celui qui joue à des fins de divertissement ou d'évasion (en recherchant par exemple la meilleure immersion possible) ou celui qui appréhende le jeu vidéo en tant que forme artistique ou discursive (en s'intéressant alors à des jeux comme Rez ou Shadow of the Colossus [Team Ico, 2005] ou au jeu indépendant ou sérieux). Il y a aussi des différences d'attentes entre le joueur passionné (hardcore gamer) et le joueur occasionnel (casual gamer). Dans le cadre d'une expérience de jeu en solo, alors que le 
joueur passionné peut être attiré par le photoréalisme ou des graphismes toujours à la fine pointe, mais qui ne le distraient pas de l'expérience (immersive) du jeu, le joueur occasionnel peut accorder une moindre importance à la qualité des graphismes s'il joue uniquement dans le but de passer un bon moment. Dans ce dernier cas, la qualité des graphismes importe moins que la transparence de l'interface, qui doit être intuitive et facile à prendre en main (ceci peut expliquer en partie le succès de la Nintendo Wii).

$\mathrm{Au}$-delà de ces aspects intrinsèques, plusieurs agents externes doivent également être pris en compte, tels que des facteurs transmédiatiques, qui concernent les influences qu'ont les autres médias sur le jeu vidéo. La principale influence est évidemment celle du cinéma, mais le jeu vidéo est aussi influencé visuellement par d'autres médias populaires, tels que la bande dessinée (Taylor, 2004), ou même par les arts visuels, comme pour le jeu Rez, alors que le créateur Tetsuya Mizuguchi s'est inspiré de l'art abstrait, plus précisément de l'œuvre de Wassily Kandinsky (Abbott, 2007). Au Japon, l'influence du manga et de l'anime se fait sentir depuis les tout débuts, contribuant à l'omniprésence de la culture visuelle populaire au Japon, dont les jeux vidéo y furent immédiatement associés et intégrés (Kohler, 2005 ; Steinberg, 2015). De ce fait, les facteurs transnationaux affectent aussi les styles audiovisuels, principalement par l'influence de la culture visuelle japonaise sur l'Europe et l'Amérique du Nord (Kelts, 2006).

Ainsi, une étude attentive des styles esthétiques du jeu vidéo montre bien qu'une lecture téléologique des graphismes du jeu vidéo est réductrice et ne peut prendre en compte les choix conscients des concepteurs de jeux. Comme partie intégrante de l'esthétique vidéoludique en général, l'aspect visuel du jeu vidéo est essentiel afin de mieux comprendre le jeu vidéo comme tel: sa structure, sa conception, et l'expérience du joueur. Il est en relation directe avec l'aspect spécifique du jeu vidéo, à savoir son interactivité.

\section{Le jeu vidéo comme art interactif : spécificités et potentialités}

Par son intérêt marqué dans les études sur les nouveaux médias, l'interactivité semble une notion associée d'emblée aux technologies numériques. Par contre, l'idée d'un système ouvert, d'une interaction plus ou moins libre entre une œuvre et son spectateur, a commencé à se manifester bien avant l'arrivée des systèmes informatiques. Déjà, au début du XXe siècle, le travail de Marcel Duchamp remettait en cause la validité de l'acte même de représentation, avec son couple dichotomique sujet-objet. L'artiste devint dès lors, non plus uniquement un créateur, mais aussi, et surtout, un « opérateur ».

Désormais, la fonction d'un artiste est souvent de gérer un réseau d'informations : « il la présente, l'organise, la compile, en cherchant parfois, mais pas toujours, à lui donner un sens. La forme de l'œuvre est donc ici dans un processus, une dynamique de correspondance » (Samper, 2003). Ainsi, l'interactivité entre l'utilisateur et la machine se veut l'aboutissement $\mathrm{du}$ processus de remise en cause de la dichotomie sujet (contemplateur) - objet (créé) dans la représentation, voire de la critique de la représentation elle-même qui a débuté au début du $\mathrm{XX}^{\mathrm{e}}$ siècle.

Par contre, comme le note Samper, les problématiques autour de la notion de représentation n'ont pas disparu. Le développement des technologies informatiques les 
remet à l'ordre du jour, mais d'une manière différente, alors que le jeu vidéo est au cœur de ce changement.

On assiste, en effet, à l'association d'outils de représentation du sensible multimédia - et d'outils issus des sciences techniques et humaines servant à modéliser les réseaux d'interactions du réel - psychologie, éthologie, mécanique, urbanisme, économie, etc. Les jeux vidéo, dans leur ensemble, peuvent être vus comme l'extension contemporaine la plus aboutie de ce processus, comme en font foi les œuvres de Will Wright (Sim City, The Sims, Spore) (Samper 2003).

Aussi immergés dans un jeu vidéo que nous pouvons l'être, nous ne sommes néanmoins jamais dans un monde réel, mais face à des simulations et des représentations des véritables systèmes d'interactivité constitutifs du réel. Cette idée est justement ce qu'on entend par interactivité dans le cadre du multimédia. Les jeux vidéo, bien qu'essentiellement interactifs, commencent seulement à proposer des interactions physiques semblables à la manière dont nous pouvons interagir sur les choses qui nous entourent. Pour l'instant, mais ceci est peut-être amené à changer sous peu, la grande lacune du jeu vidéo est encore le toucher (quoiqu'il semble constituer dans le domaine haptique un pas de géant par rapport au cinéma). ${ }^{13}$ Le joueur utilise bel et bien ses mains, mais seulement parce qu'il se projette dans l'action exécutée à l'écran, et non en réponse à une sensation physique complexe qu'il pourrait éprouver, comme c'est le cas avec le jeu virtuel dépeint dans le film eXistenZ (Cronenberg, 1999). Cependant, de véritables efforts sont accomplis dans ce domaine par les développeurs de matériels informatiques et vidéoludiques afin de créer des interfaces haptiques. L'arrivée en triomphe de la console Wii de Nintendo, avec sa manette à senseur, était déjà un pas en ce sens, c'est-à-dire une étape vers la réalisation des fantasmes des films de science-fiction représentant des systèmes de réalité virtuelle idéaux. Au moment d'écrire ces lignes, il reste encore à voir si les nouveaux espoirs de la réalité virtuelle d'aujourd'hui répondront à ces vieux fantasmes.

Si les interactions dans le jeu vidéo sont de simples simulations de véritables systèmes d'interactivité réels, quelle est sa spécificité au niveau de ses interactions ? Samper distingue en ce sens deux types d'interaction à l'œuvre: l'interactivité objective et l'interactivité subjective (Samper 2003). ${ }^{14}$ L'interactivité objective est le type d'interactivité utilisé dans le jeu vidéo depuis ses origines, en la scénarisant plus ou moins. C'est l'interactivité que l'on voit dans toutes les formes de simulation de modèles physiques ou mécaniques, par exemple les simulations d'automobiles, d'avions, de trains, de ski, de pêche, de cuisine, etc. Toutefois, les simulations peuvent aussi être plus complexes et protéiformes, comme c'est le cas avec Sim City (Maxis, 1989) qui simule à la fois une économie, une écologie et un aménagement urbain. Grâce à l'interface, le lien intangible qui pouvait traditionnellement exister entre l'œuvre et le spectateur se concrétise dans les œuvres informatiques et le jeu vidéo. Le joueur, ou l'utilisateur, peut provoquer des actions à l'intérieur de l'œuvre, qui réagira en conséquence. Dans le jeu vidéo, l'interactivité se trouve en symbiose avec le gameplay.

Le "game» (le jeu circonscrit et régulé) et le "play» (le jeu libre et créatif) sont des termes qui permettent de décrire l'activité principale derrière le jeu vidéo, sa jouabilité (Perron, 2003). Néanmoins, la spécificité du jeu vidéo, à l'extérieur du champ des études vidéoludiques, est davantage rattachée à l'interactivité, terme extrêmement populaire depuis déjà plus d'une décennie. Contrairement aux romans, aux pièces de théâtre ou aux films, où un auteur (actif) s'adresse au lecteur/spectateur (inactif, bien que non passif), le discours académique sur l'interactivité stipule fréquemment que le joueur ${ }^{15}$ est 
immédiatement placé au cœur de l'expérience néomédiatique et qu'il devient ainsi un agent actif des évènements narratifs ou formels. Pour certains, de manière plutôt exagérée faut-il ajouter, il devient même en quelque sorte co-auteur ou co-créateur du récit.

Toutefois, la dérive de l'autorité auteuriale (Barthes annonçait la « mort de l'auteur » il y a plus de 30 ans), par l'utilisation non critique de la notion d'interactivité dans une variété de contextes très différents, que ce soit pour les jeux vidéo, le support DVD, les " reality shows » de la télévision, ou même par une révision de l'activité du spectateur dans les médias traditionnels, ${ }^{16} \mathrm{a}$ occasionné une compréhension très large (et floue) du concept. Au début des études sur les univers ludiques (Aarseth, 1997 et Manovich, 2001), l'ambiguïté du concept d'interactivité exigeait l'emploi d'un meilleur terme pour décrire ce phénomène. Aarseth (1997) proposa le terme "ergodicité », emprunté à la physique, afin de rendre compte de l'activité non triviale du cybertexte. Jesper Juul (2005), de son côté, suggérait que le terme ne devait pas être écarté, car il décrivait bien l'activité spécifique de la rencontre humain-machine dans le jeu vidéo: "Les jeux vidéo sont interactifs parce que les actions du joueur jouent un rôle sur le déroulement des événements dans le jeu » (Juul, 1999, p. 21). Selon Gonzalo Frasca $(1999$; 2003) ou Janet Murray (1997), l'interactivité du jeu vidéo se remarquait dans la possibilité pour le joueur d'opérer une transformation sur le jeu (en tant que «texte ») à l'aide de manipulations, durant sa performance (play), en accord avec l'imposition des règles du jeu (game). Janet Murray nommait cet échange conversationnel "pouvoir d'action» (agency) (Murray, 1997, p. 126-153).

Pour Chris Crawford (1984), c'est ce type d'interactivité offerte par le jeu vidéo qui permet de le différencier d'un jeu plus statique comme le casse-tête. Car le jeu vidéo, cette fois en tant que simulation, répond aux efforts et à l'activité du joueur. De même, cette interactivité est différente de celle offerte par le support DVD ou une émission de télévision interactive, puisque dans ces derniers aucun potentiel transformatif n'est permis. Comme le note James Newman : «Sélectionner une option parmi d'autres permet au spectateur d'un DVD de modifier l'ordre, la temporalité ou de parcourir aisément le materiel, mais pas d'altérer le contenu substantiel des séquences" (Newman, 2004, p. 26-27). Marie-Laure Ryan (2001), en continuité avec Janet Murray (1997) et Brenda Laurel (1991), va indiquer que le jeu vidéo peut s'adapter de façon dynamique à la performance du joueur. Ceci est possible, car son contenu n'est pas fixe, mais procédural. Grâce à son moteur de jeu travaillant en temps réel, le jeu vidéo est orienté autour d'une simulation transformable et réceptive.

Ainsi, le jeu vidéo se différencie des médias traditionnels, tels que le cinéma, par son interactivité (qui sous-tend l'interaction humain-machine à travers une interface particulière $)^{17}$ et son aspect ludique, mais se distingue également des autres types de jeux - par son pouvoir d'action permis par la machine procédurale - et des autres médias interactifs. Par contre, Newman (2004) insiste sur le fait que la discussion autour de la notion d'interactivité dans le jeu vidéo demeure problématique alors que certains aspects néanmoins essentiels du jeu vidéo, tels que la cinématique, sont exempts d'interactivité. L'expérience du jeu vidéo est le résultat d'éléments (interactifs ou non) très variés où chaque aspect offre des degrés d'activité et exige des types de participation distincts.

Peu importe la définition qu'on en donne, ${ }^{18}$ en examinant son lien avec l'art, c'est possiblement ce caractère interactif qui pourrait justement permettre au jeu vidéo de se positionner comme «nouvel » art. C'est entre autres ce qu'avançait déjà Boulon en 2008 
en mentionnant que le jeu vidéo pouvait réussir à s'émanciper comme pratique artistique un utilisant sa spécificité interactive pour créer des œuvres transcendant la simple fonctionnalité du médium par une recherche de sens et de significations plus profondes, et qui surtout viendraient questionner la nature même du médium ou de la notion d'art, un positionnement " méta » qui a marqué l'art du XXe siècle. Toutefois, même sans cette démarche artistique contemporaine, le jeu vidéo est de plus en plus associé à l'art, et tend peu à peu à s'institutionnaliser, même dans les marchés de l'art, ne serait-ce que par l'émergence du game art (œuvres d'art inspirées du jeu vidéo) et des art games (jeux vidéo créés en tant qu'œuvres d'art), ou par la présence de plus en plus marquée du jeu vidéo dans les musées (Bittanti et Quarenta, 2006 ; Kelman, 2006 ; Clarke, 2007). Même si le débat sur la qualité artistique du jeu vidéo n'a sûrement pas fini d'attiser les passions, du moins pas tant que la dominance de sa production se situe dans le réseau commercial, il ne fait nul doute que par sa diversité de pratiques et de modes de réception, le jeu vidéo et l'art sont désormais deux entités indissociables.

\section{Annexe - Évolution des styles vidéoludiques}

Les figures ci-dessous sont le résultat d'un travail de compilation et de classement de jeux vidéo selon leurs styles tous les dix ans (sauf pour cette année), à commencer par l'année 2008, celle où la recherche fut essentiellement effectuée dans le cadre de mes études doctorales. Pour chaque année, les jeux qui n'étaient pas des originaux ou des remakes (par exemple les ports sur différentes consoles, les localisations, les extensions, le contenu téléchargeable ou les remasterisations de jeux antérieurs) furent exclus de la liste. À noter également qu'en raison de leur nombre trop élevé, les jeux ne sont pas référencés ici. Les sources consultées proviennent de bases de données de jeux proposées sur des sites tels que Mobygames, Gamefaqs, All Game Guide, et Wikipedia, de même que des sites avec du contenu vidéo tels que YouTube, Gamespot ou IGN.

Cette tâche, qui a surtout une valeur heuristique, permet de bien cerner les différents mouvements stylistiques au cours des quarante ans d'histoire du jeu vidéo. Évidemment, ceci est un premier travail de défrichage, alors que le projet pourrait prendre une plus grande ampleur en ajoutant des périodes (aux cinq ans, quatre ans, trois ans, etc.) afin de préciser les conclusions présentées précédemment.

1978 - Abstractionnnisme - Les styles visuels pour l'année 1978 furent principalement marqués par les limitations technologiques et les outils graphiques disponibles pour l'époque, alors que le style dominant fut l'abstractionnisme (Figure 12). 
Figure 12

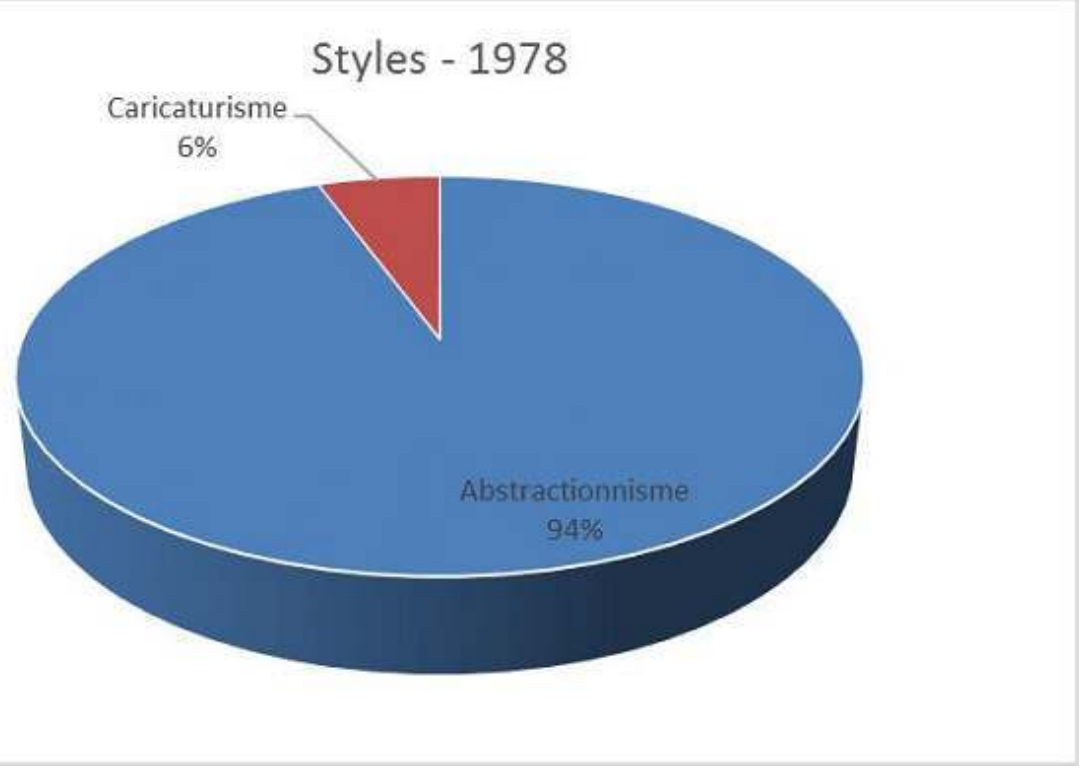

1978 - Style dominant : l'abstractionnisme. Échantillon : 18 jeux.

1988 - Caricaturisme - Les styles visuels pour l'année 1988 furent déterminés par la popularité des consoles 8-bit (Nintendo Entertainment System [1985], Sega Master System [1986]) et l'amélioration technologique des jeux d'arcade qui ont permis la popularité des graphismes caricaturaux (Figure 13).

\section{Figure 13}

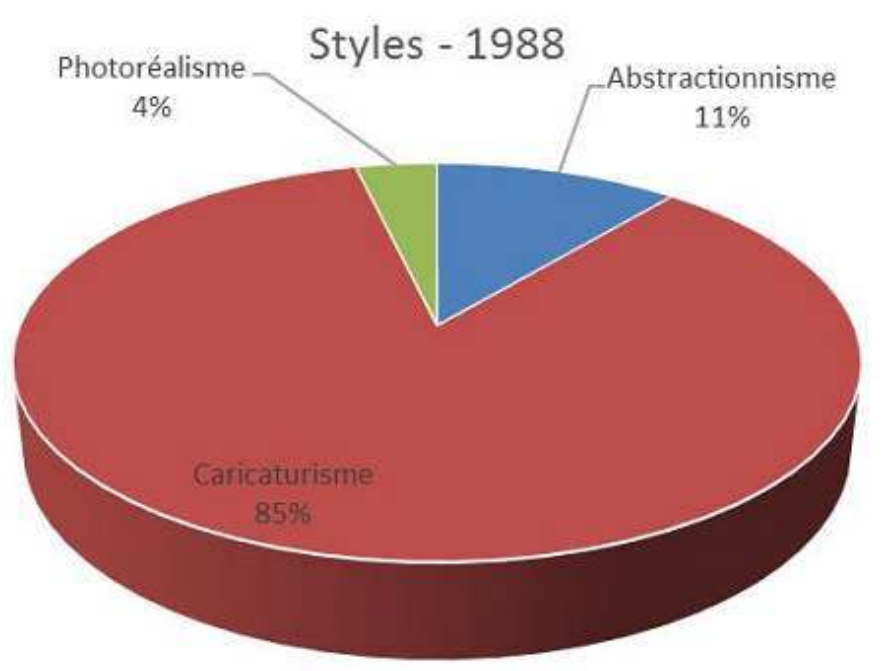

1988 - Style dominant : le caricaturisme. Échantillon : 27 jeux.

541998 - Photoréalisme - Les cartes accélératrices et les logiciels d'animation 3D permirent le rendu d'une image photoréaliste qui caractérisa le style visuel de l'année 1998 (Figure 14). 
Figure 14

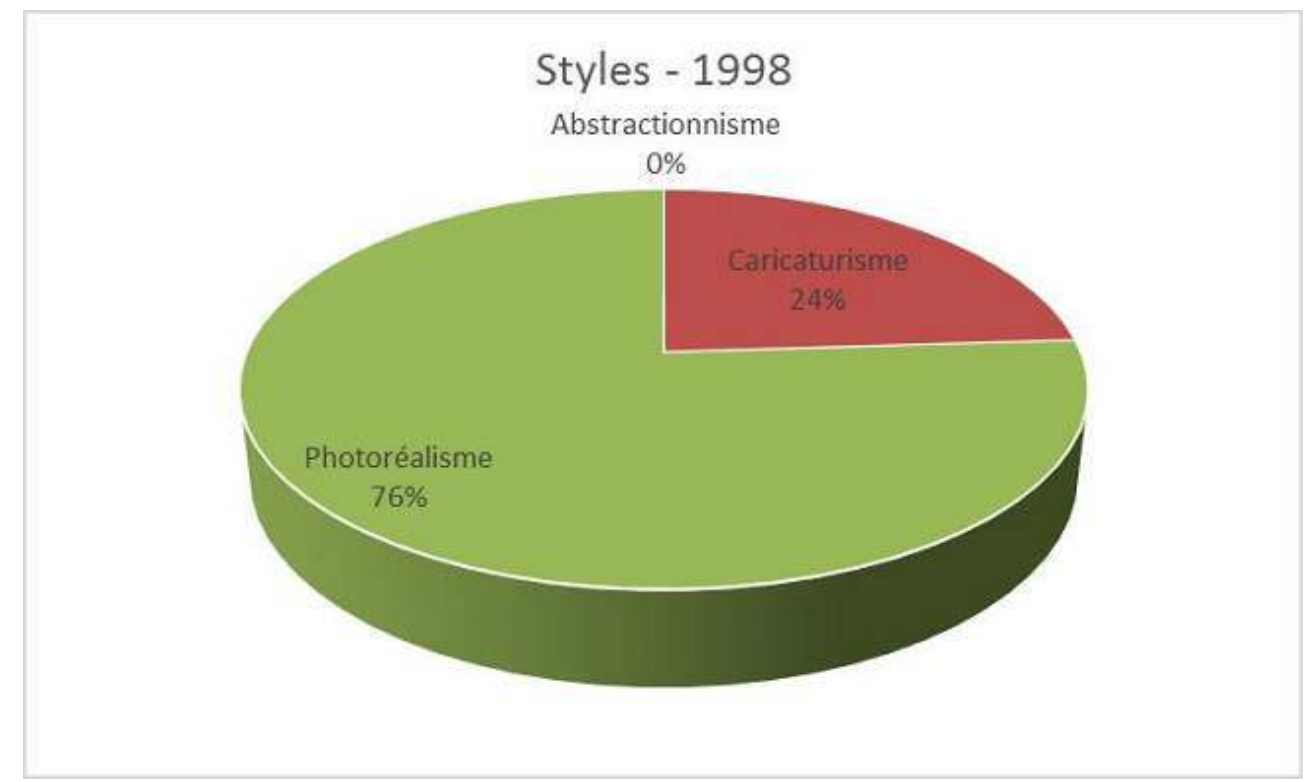

1998 - Style dominant : le photoréalisme. Échantillon : 25 jeux.

2008 - Diversité des styles - Après la saturation des jeux à tendance photoréaliste, qui dominent toujours néanmoins, un retour aux alternatives esthétiques que sont le caricaturisme et l'abstractionnisme permettent une plus grande diversité des styles (Figure 15).

\section{Figure 15}

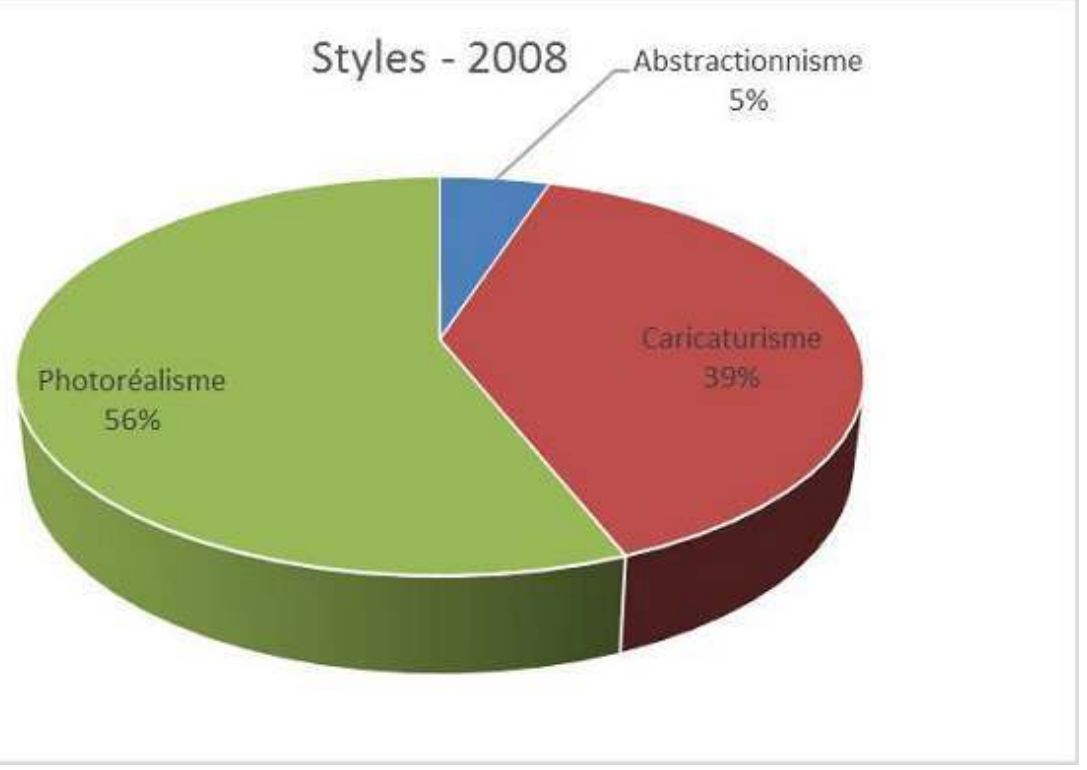

2008 - Diversité des styles. Échantillon : 100 jeux.

2016 - Retour du caricaturisme - Grâce à l'essor du jeu vidéo indépendant, et de la tendance à une certaine nostalgie pour les jeux anciens, le caricaturisme fait un retour marqué (Figure 16). La catégorisation se base sur le premier quart de l'année (jeux sortis de janvier à mars 2016). 


\section{Figure 16}

\section{Styles 2016}

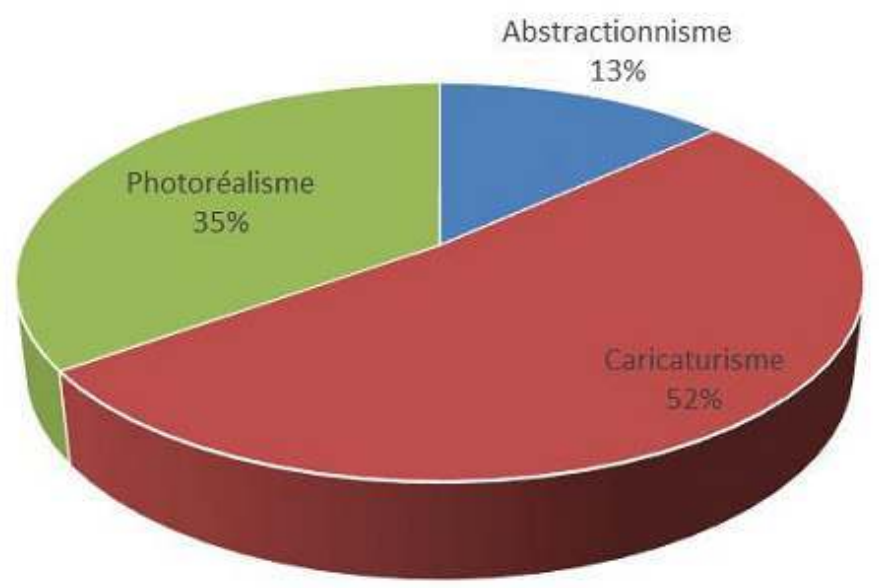

2016 - Retour du caricaturisme. Échantillon : 83 jeux.

\section{BIBLIOGRAPHIE}

AARSETH E. (1997), Cybertext. Perspectives on Ergodic Literature, Baltimore, Johns Hopkins University Press.

AARSETH E. (1998), « Allegories of Space: The Question of Spatiality in Computer Games », http:// interactive.usc.edu/blog-old/wp-content/uploads/2010/08/Allegories_of_Space.pdf

ABBOTT M. (2007), « Art by Mizuguchi », Brainy Gamer, September 20, http:// www.brainygamer.com/the_brainy_gamer/2007/09/welcome-to-the-.html ARISTOTE (1990), Poétique, traduit par Michel Magnien, Paris, Le Livre de Poche.

AOYAMA Y. et IZUSHI H. (2006), « Industry evolution and cross-sectoral skill transfers: a comparative analysis of the video game industry in Japan, the United States, and the United Kingdom », Environment and Planning A, Vol. 38, № 10, pp. 1843--1861.

ARSENAULT D. et CÔTÉ P.-M. (2013), « Reverse-engineering graphical innovation: an introduction to graphical regimes », GAME: The Italian Journal of Game Studies, № 2, http://www.gamejournal.it/ reverse-engineering-graphical-innovation-an-introduction-to-graphical-regimes/

AUMONT J., BERGALA A., MARIE M. et VERNET M. (1994), Esthétique du film, Paris, Nathan.

AUKSTAKALNIS S. (2016), Practical Augmented Reality: A Guide to the Technologies, Applications, and Human Factors for AR and VR, Indianapolis, Indiana, Addison-Wesley Professional. 
BITTANTI M. \& QUARANTA D. (dir.) (2006), GameScenes: Art in the Age of Videogames, Milan, Johan and Levi.

BLESZINSKI C. (2001), « The Art and Science of Level Design », Gamasutra, 10 janvier, http:// gamedevs.org/uploads/the-art-science-of-level-design.doc

BOLTER J. D. \& GRUSIN R. (1999), Remediation: Understanding New Media, Cambridge, Mass., MIT Press.

BORDWELL D. \& CARROLL N. (1996), Post-Theory: Reconstructing Film Studies, Madison, University of Winconsin Press.

BOTTOMLEY P. (2011), Do Technical Limitations Hinder Creativity?, mémoire de maîtrise, University of Central Lancashire, https://petebottomley.wordpress.com/do-technicallimitations-hinder-creativity-part-1/

BOULON O. (2008), « Le jeu vidéo est-il un art? », Canard PC, Numéro 167, Mars.

CAMPER B. (2009), « Retro Reflexivity: La-Mulana, an 8-Bit Period Piece », in Perron B. \& Wolf M.J.P. (eds.), The Video Game Theory Reader 2, London/New York, Routledge, pp. 169-195.

CLARKE A. (ed.) (2007), Videogames and Art, Bristol, UK, Intellect.

COLLINS K. (ed.) (2008a), From Pac-Man to Pop Music: Interactive Audio in Games and New Media, London/New York, Routledge.

COLLINS K. (2008b), Game Sound: An Introduction to the History, Theory, and Practice of Video Game Music and Sound Design, Cambridge, Mass., The MIT Press.

COLLINS K. (2013), Playing with Sound: A Theory of Interacting with Sound and Music in Video Games, Cambridge, Mass., The MIT Press.

COMETTI J.P., MORIZOT J. et POUIVET R. (dir.) (2005), Esthétique contemporaine : Art, représentation et fiction, Paris, Vrin.

CRAWFORD C. (1984), The Art of Computer Game Design, San Francisco, Osbourne-McGraw-Hill.

DARLEY A. (2000), Visual Digital Culture: Surface Play and Spectacle in New Media Genres, London/New York, Routledge.

EBERT R. (2010), « Video games can never be art », rogerebert.com, 16 avril, http:// www.rogerebert.com/rogers-journal/video-games-can-never-be-art

EGENFELDT-NIELSEN S., SMITH J.H. \& TOSCA S. (2008), Understanding Video Games: The Essential Introduction, London/New York, Routledge.

FRASCA G. (1999), « Ludology Meets Narratology: Similitude and Difference Between (Video)games and Narrative », http://www.ludology.org/articles/ludology.htm

FRASCA G. (2003), « Simulation versus Narrative: Introduction to Ludology », in Wolf M.J.P.\& Perron B. (dir.), The Video Game Theory Reader, London/New York, Routledge, pp. 221-235.

GENVO S. (2003), Introduction aux enjeux artistiques et culturels des jeux vidéo, Paris, L'Harmattan. GERVEREAU L.(2000), Histoire du visuel au XXe siècle, Paris, Seuil.

GOMBRICH E.H. (2002), L'art et l'illusion: Psychologie de la représentation picturale [1960], Paris, Phaidon.

GRAU O. (2003), Virtual Art: From Illusion to Immersion, Cambridge, Mass., The MIT Press. 
GRODAL, T. (2003), « Stories for Eye, Ear, and Muscles: Video Games, Media, and Embodied Experiences », in Wolf M.J.P.\& Perron B. (dir.), The Video Game Theory Reader, London/New York, Routledge, pp. 129-155.

GUENZEL S. (2014), Push Start: The Art of Video Games, Hamburg, Germany, Earbooks.

GÜNZEL S. (2008), « The Space-Image Interactivity and Spatiality of Computer Games », in Günzel S., Liebe M. et Mersch D. (dir.), Conference Proceedings of the Philosophy of Computer Games 2008, Postdam, Potsdam University Press, pp. 170-189.

HAMUS-VALLÉE R. (2004), Les effets spéciaux, Paris, Les Cahiers du cinéma.

HEGEL G.W.F. (1953), L’Esthétique, textes choisis par Claude Khodoss, Paris, Presses Universitaires de France.

HAYWARD D. (2005), « Videogame Aesthetics: The Future! », Gamasutra.com, 14 octobre, http:// www.gamasutra.com/features/20051014/hayward_01.shtml

HUMBLE R. (2006), « Game Rules as Art », The Escapist, 18 avril, http:// www.escapistmagazine.com/articles/view/video-games/issues/issue_41/247-Game-Rules-as-Art IWATA ASKS (2009), « New Super Mario Bros. Wii », Volume 1 : Shigeru Miyamoto, 13 novembre, http://iwataasks.nintendo.com/interviews/\#/wii/nsmb/0/0

JÄRVINEN A. (2002), « Gran Stylissimo: The Audiovisual Elements and Styles in Computer and Video Games », in Mäyrä F. (dir.), Proceedings of Computer Games and Digital Cultures Conference, Tampere, Tampera University Press, pp. 113-128.

JENISCH J. (2008), The Art of the Video Game, Philadelphia, PA, Quirk Books.

JENKINS H. et SQUIRE K.D. (2002), « The Art of Contested Spaces », in King L. (dir.), Game On: The History and Culture of Videogames, London, Barbican Press, pp. 64-75.

JIMENEZ M. (1997), Qu'est-ce que l'esthétique ?, Paris, Gallimard.

JIMENEZ M. (1999), L'esthétique contemporaine : tendances et enjeux, Paris, Klincksieck.

JIMENEZ M. (2005), La querelle de l'art contemporain, Paris, Gallimard.

JULLIER L. (1998), Les images de synthèse, Paris, Nathan.

JUUL J. (1999), A Clash between Game and Narrative: A Thesis on Computer Games and Interactive Fiction, thèse de doctorat, Université de Copenhague.

JUUL J. (2005), Half-Real: Video Games Between Real Rules and Fictional Worlds, Cambridge, Mass., The MIT Press.

KANT E. (1990), Observations sur le sentiment du beau et du sublime, traduit par Monique DavidMénard, Paris, GF-Flammarion.

KANT E. (1995), Critique de la faculté de juger, traduit par Alain Renaut, Paris, GF-Flammarion.

KELMAN N. (2006), Video Game Art, New York, Assouline Publishing.

KELTS R. (2006), Japanamerica: How Japanese Pop Culture Has Invaded the U.S., New York, St-Martin's Press.

KING G. \& KRZYWINSKA T. (dir.) (2002), Screenplay: Cinema/Videogames/Interfaces, London/New York, Wallflower Press.

KOHLER C. (2005), Power-Up: How Japanese Video Games Gave the World an Extra Life, Indianapolis, Indiana, Brady Games. 
LAMARQUE P. (2004), « Reflections on Current Trends in Aesthetics », Postgraduate Journal of Aesthetics, Vol. 1, № 1, Avril, pp. 1-9.

LANDAY L. (2014), « Interactivity », in Wolf M.J.P.\& Perron B. (dir.), The Routledge Companion to Video Game Studies, New York, Routledge, pp. 173-184.

LASSETER J. (1987), « Principles of Traditional Animation Applied to 3D Computer Animation », Computer Graphics, Vol. 21, № 4, Juillet, pp. 35-44.

LAUREL B. (1991), Computers as Theatre, Reading, Mass., Addison-Wesley Publishing.

LE DIBERDER A. et LE DIBERDER F. (1993), Qui a peur des jeux vidéo?, Paris, La Découverte.

LE DIBERDER A. et LE DIBERDER F. (1998), L'univers des jeux vidéo, Paris, La Découverte.

LEROUX Y. (2016), « La littératie vidéo ludique », Psychologik, 20 mars, http://

psychologik.blogspot.de/2016/03/la-litteratie-video-ludique.html

LISTER M., DOVEY J., GIDDINGS S., GRANT I. \& KELLY K. (2003), New Media: A Critical Introduction, London/New York, Routledge.

LUNDGREN S., BERGSTRÖM K. J. \& BJÖRK S. (2009), « Exploring Aesthetic Ideals of Gameplay », Breaking New Ground: Innovation in Games, Play, Practice and Theory, DiGRA Proceedings, http:// www.digra.org/dl/db/09287.58159.pdf

MANOVICH L. (2001), The Language of New Media, Cambridge, Mass., The MIT Press.

MCCLOUD S. (1993), Understanding Comics : The Invisible Art, New York, HarperCollins.

MCLAUGHLIN, M.L., HESPARINA J.P. \& SUKHATME G.S. (dir.) (2002), Touch in Virtual Environments: Haptics and the Design of Interactive Systems, New Jersey, IMSC Press.

MELISSINOS C. et O'ROURKE P. (2012), The Art of Video Games: From Pac-Man to Mass Effect, New York, Welcome Books.

MURRAY J.H. (1997), Hamlet on the Holodeck : the Future of Narrative in Cyberspace, Cambridge, Mass., The MIT Press.

NEWMAN J. (2004), Videogames, London/New York, Routledge.

NIEDENTHAL S. (2008), Complicated Shadows: The Aesthetic Significance of Simulated Illumination in Digital Games, thèse de doctorat, Blekinge Institute of Technology.

NIEDENTHAL S. (2009), « What We Talk About When We Talk About Game Aesthetics », Breaking New Ground: Innovation in Games, Play, Practice and Theory, DiGRA Proceedings, http:// www.digra.org/dl/db/09287.17350.pdf

OCHELlA B. (2007), « Are Games Art? (Here We Go Again...) », Gamasutra, 16 mars, http:// www.gamasutra.com/view/feature/130113/are_games_art_here_we_go_.php

PARKER F. (2013), « An Art World for Artgames », Loading... The Journal of the Canadian Game Studies Association, Vol. 7, № 11, pp. 41-60.

PARKER F. (2014), « Infinite Typewriters: Canon, Criticism, and BioShock », First Person Scholar, 19 février, http://www.firstpersonscholar.com/infinite-typewriters/

PERRON B. (2003), « From Gamers to Players and Gameplayers: The Example of Interactive Movies ", in Wolf M.J.P.\& Perron B. (dir.), The Video Game Theory Reader, London/New York, Routledge, pp. 237-258. 
PERRON B. et THERRIEN C. (2009), « De la "sortie de Spacewar! des laboratoires de MIT" à Gears of War, ou comment l'image vidéoludique est devenue plus cinématographique », bianco e nero, № 564, mai-août, pp. 40-50.

PIERSON M. (1999), «CGI effects in Hollywood science-fiction cinema 1989-95 : the wonder years », Screen, Vol. 40, № 2, Summer, pp.158-176.

POOLE S. (2000), Trigger Happy: Videogames and the Entertainment Revolution, New York, Arcade Publishing.

RICKITT R. (2000), Special Effects: The History and Technique, New York, Billboard Books.

RYAN M.-L. (2001), Narrative as Virtual Reality: Immersion and Interactivity in Literature and Electronic Media, Baltimore, The Johns Hopkins University Press.

SAMPER B. (2003), « Les artistes et le jeu vidéo », Leonardo/Olats, mars, http://www.olats.org/ projetpart/artmedia/2002/t_bSamper.html

SHERRINGHAM M. (2003), Introduction à la philosophie esthétique [1992], Paris, Payot et Rivages.

SOMMERSETH H. (2009), « Exploring Game Aesthetics », Breaking New Ground: Innovation in Games, Play, Practice and Theory, DiGRA Proceedings, http://www.digra.org/dl/db/09287.58198.pdf

STEINBERG M. (2015), « 8-Bit Manga: Kadokawa's Madara, or, The Gameic Media Mix », Kinephanos, Vol 5 (Geemu and media mix: Theoretical approaches to Japanese video games / Geemu et media mix : Approches théoriques du jeu vidéo japonais), pp. 40-52, http:// www.kinephanos.ca/2015/8-bit-manga/

STUART K. (2014), « Video games and art: why does the media get it so wrong? », The Guardian, 8 janvier, http://www.theguardian.com/technology/gamesblog/2014/jan/08/video-games-artand-the-shock-of-the-new

TAVINOR G. (2009), The Art of Videogames, Malden, MA, Wiley-Blackwell.

TAYLOR L.N. (2004), « Compromised Divisions : Thresholds in Comic Books and Video Games », ImageTexT : Interdisciplinary Comics Studies, Vol. 1, № 1, printemps, http://www.english.ufl.edu/ imagetext/archives/v1_1/taylor/

THOMAS F. et JOHNSTON O. (1981), The Illusion of Life: Disney Animation, New York, Disney Editions. VON BORRIES F., WALZ S.P. \& BÖTTGER M. (eds.) (2007), Space, Time, Play: Computer Games, Architecture and Urbanism: The Next Level, Berlin, Birkhãuser.

WARREN J. (2011), « Game designers want to be artists without knowing what that means », Kill Screen, 21 mars, https://killscreen.com/articles/game-designers-want-be-artists-withoutknowing-what-means/

WOLF M.J.P. (2001), The Medium of the Video Game, Austin, University of Texas Press.

WOLF M.J.P. (2003), « Abstraction in the Video Game », in Wolf M.J.P. \& Perron B. (eds.), The Video Game Theory Reader, London/New York, Routledge, pp. 47-65.

WOLF M.J.P. (2012), « video games as art », in Wolf M.J.P. (ed.), Encyclopia of Video Games: The Culture, Technology, and Art of Gaming, Santa Barbara, Greenwood Press.

ZAGAL J.P. (2009), « A Framework for Games Literacy and Understanding Games », Loading, Vol 3, № 5, pp. 1-16, http://journals.sfu.ca/loading/index.php/loading/article/view/74/67 


\section{NOTES}

1. Voir entre autres Cometti, Morizot et Pouivet (2005), Jimenez (1999) et (2005), et Lamarque (2004).

2. Au sujet de l'exploration et la conquête de l'espace vidéoludique, voir également Jenkins et Squire (2002).

3. Déjà, en 1993, les frères Le Diberder parlaient du jeu vidéo comme le dixième art (Le Diberder et Le Diberder 1993, 1998). Depuis, le débat a refait surface plus d'une fois. Voir, entre autres, Ochalla (2007), Boulon (2008), Ebert (2010), Warren (2011) et Stuart (2014).

4. Pour ne nommer que quelques ouvrages généraux (alors qu'il s'en est publié davantage sur des jeux particuliers) : Jenisch (2008), Melissinos et O'Rourke (2012), Guenzel (2014).

5. Voir à ce sujet les ouvrages généraux de Sherringham $(1992,2006)$ et Jimenez (1997). Dans le cadre plus précis du lien entre le jeu vidéo et l'art institutionnalisé, voir Warren (2011).

6. Pour preuve, il suffit d'observer le développement des images de synthèse où la technologie a eu une incidence directe sur son aspect visuel (Pierson, 1999 ; Rickitt, 2000 ; Hamus-Vallée, 2004).

7. Sur le lien entre jeu vidéo et architecture, voir Von Borries, Walz et Böttger (2007).

8. Une étude de l'audiovisualité du jeu vidéo devrait également inclure son aspect sonore, mais vu l'ampleur que prendrait une telle tâche, je me dois de négliger cette question ici, qui comprend de toute façon son lot de littérature spécifique. Voir entre autres le travail de Karen Collins (2008a, 2008b, 2013).

9. Bien entendu, les perspectives narratologiques et ludologiques ne définissent pas l'ensemble des approches de l'étude du jeu vidéo, comme le mentionne Stephan Günzel en indiquant un «tournant spatial » (spatial turn) des études vidéoludiques durant les années 2000 (Günzel, 2008, p. 170-171).

10. La théorie des auteurs est tout de même de plus en plus abordée dans le champ des études vidéoludiques, voir par exemple le travail de Felan Parker $(2013 ; 2014)$.

11. L'exagération est le dixième principe de l'animation selon Thomas et Johnston (1981, p. 65-66). Voir aussi Lasseter (1987, p. 41) pour une application de ce principe à l'animation numérique.

12. De Tron (Steven Lisberger) et Star Trek II : The Wrath of Khan (Nicholas Meyer) en 1982 aux premiers courts métrages de John Lasseter chez Pixar (The Adventures of Andre and Wally B. [1984], Luxo Jr. [1986]).

13. Voir les changements majeurs de ces technologies depuis les quinze dernières années, de McLaughlin, Hesparina et Sukhatme (2002) à Aukstakalnis (2016), en passant par la Springer Series on Touch and Haptic Systems : http://www.springer.com/series/8786.

14. L'interactivité subjective est la représentation des processus qui déterminent les échanges sociaux, tels qu'on les rencontre dans les jeux en ligne.

15. Plusieurs termes furent d'ailleurs utilisés pour tenter de décrire cette nouvelle position: « utilisateur », « viewser », « interacteur », « spectActeur » ou simplement « joueur » (« player » ou «gamer»).

16. Voir Manovich (2001). Cette approche découle bien entendu de l'influence des études cognitives sur le cinéma depuis le milieu des années 1980, dont David Bordwell et Noël Carroll en furent les principaux représentants (Bordwell et Carroll, 1996).

17. Le développeur de jeu Rod Humble, créateur de The Marriage, va explorer l'idée des règles de jeu en tant qu'art (Humble, 2006).

18. Depuis ces réflexions, le concept a perdu de l'intérêt dans les études du jeu vidéo. Pour un survol plus détaillé à propos de la notion d'interactivité en lien avec le jeu vidéo, voir Landay (2014). Elle souligne entre autres trois approches majeures dans la définition de l'interactivité qui 
ont émergé dans le champ des études sur les nouveaux médias : 1) celles qui se concentrent sur les systèmes et la technologie; 2) celles qui abordent l'expérience des utilisateurs dans ses processus d'échanges et de réponses; 3 ) celles qui s'intéressent aux activités, comportements et perceptions des utilisateurs.

\section{RÉSUMÉS}

Dans cet article, l'auteur questionne le lien complexe entre le jeu vidéo et l'art en tentant de réfléchir à l'esthétique du jeu vidéo. Selon lui, le questionnement sur la qualité artistique du jeu vidéo repose principalement sur une confusion dans la définition de la notion d'art. Trois conceptions différentes semblent être en cause dans le discours général : le jeu vidéo comme production ou technique artistique, le jeu vidéo comme média audiovisuel, et le jeu vidéo comme pratique interactive. En distinguant à tour de rôle ces trois acceptions, l'auteur se penche également sur la question des styles visuels de jeu vidéo, alors que ces derniers contribuent de manière primordiale à la perception du jeu vidéo en tant qu'objet esthétique et culturel.

In this article, the author questions the complex relationship between video game and art in trying to think about the aesthetics of video games. According to him, the questioning of the artistic quality of the medium is mainly based on a confusion in the definition of the concept of art. Three different significations seem to be involved in the general discourse: video game as an artistic production or technique, video game as an audiovisual medium, and video game as an interactive practice. While distinguishing between the three meanings, the author also addresses the issue of video game visual styles, since they contribute significantly to the perception of video game as an aesthetic and cultural object.

\section{INDEX}

Mots-clés : jeu vidéo, esthétique, art, style, interactivité

Keywords : video game, aesthetics, interactivity

\section{AUTEUR}

\section{MARTIN PICARD}

Université de Leipzig 